\title{
Asymptotic Behavior of the Hyperbolic Schwarz Map at Irregular Singular Points
}

\author{
By \\ Tatsuya KoIKE ${ }^{1}$, Takeshi SASAKI ${ }^{2}$ and Masaaki YoshIDA ${ }^{3}$ \\ (Kobe University ${ }^{1,2}$ and Kyushu University ${ }^{3}$, Japan)
}

\begin{abstract}
Geometric study of a second-order Fuchsian differential equation $u^{\prime \prime}-q(x) u=0$, where $q$ is rational in $x$, has been made via the Schwarz map as well as via the hyperbolic and the derived Schwarz maps ([SYY]). When the equation admits an irregular singularity, such a study was first made in $[\mathrm{SY}]$ treating the confluent hypergeometric equation and the Airy equation. In this paper, we study the hyperbolic Schwarz map (note that this map governs the other Schwarz maps) of such an equation with any irregular singularity. We describe the asymptotic behavior of the map around the singular point: when the Poincare rank is generic, it admits a uniform description; when the Poincaré rank is exceptional, a detailed study is made.

Key Words and Phrases. Hyperbolic Schwarz map, Irregular singularity, Asymptotic behavior.
\end{abstract}

2000 Mathematics Subject Classification Numbers. 33C05, 53C42.

\section{Contents}

$\begin{array}{ll}\text { 1. Introduction } & 100\end{array}$

2. Formal solutions and asymptotic expansions 101

2.1. Construction of formal solutions 101

2.2. Asymptotic expansions of solutions 103

$\begin{array}{ll}\text { 2.3. Stokes multipliers } & 107\end{array}$

3. Asymptotic behavior of the hyperbolic Schwarz map 109

3.1. Preparation of the estimates of the hyperbolic Schwarz map 109

3.2. A detailed estimate for the case $n=0 \quad 110$

3.3. The case $n=1$, a generalization of Airy differential equation 114

3.4. The case $n=-1 \quad 116$

3.5. The curve $\operatorname{Re} \Lambda(x)=c \quad 117$

3.6. The case $n \geq 1 \quad 119$

3.7. Summary 120

4. Analytic continuation of hyperbolic Schwarz map 120

$\begin{array}{ll}\text { 4.1. General case } & 121\end{array}$

4.2. Example: the Airy equation 124

4.3. Example: the equation with $q(x)=x^{n} \quad 126$ 
A. Determination of Stokes multipliers

A.1. Some relations of Stokes multipliers for $q(x)=x^{n}$

A.2. Stokes multipliers for $q(x)=x^{n}$

References

\section{Introduction}

For an equation of the form

$$
u^{\prime \prime}-q(x) u=0
$$

defined on a domain in the complex plane with coordinate $x$ with $q$ a rational function in $x$, we defined in $[\mathrm{SYY}]$ the hyperbolic Schwarz map

$$
\mathscr{S}: x \mapsto U(x)^{t} \bar{U}(x) \in \mathbf{H}^{3}, \quad U=\left(\begin{array}{cc}
u_{0} & u_{0}^{\prime} \\
u_{1} & u_{1}^{\prime}
\end{array}\right) .
$$

Here, $\mathbf{H}^{3}$ denotes the hyperbolic 3-space identified with the space $\operatorname{Her}^{+}(2)$ of positive-definite hermitian $2 \times 2$-matrices modulo positive reals, and $\left\{u_{0}, u_{1}\right\}$ are linearly independent solutions of $(E)$. Let $L_{1}=\left\{\left(x_{0}, x_{1}, x_{2}, x_{3}\right) \mid x_{0}^{2}-x_{1}^{2}-x_{2}^{2}-\right.$ $\left.x_{3}^{2}=1\right\}$ be the hypersphere in the Lorenz-Minkowski 4-space. Then, we identify $\mathbf{H}^{3}$ with the 3 -ball $\mathbf{B}^{3}=\left\{\left(y_{1}, y_{2}, y_{3}\right) \mid y_{1}^{2}+y_{2}^{2}+y_{3}^{2}<1\right\}$ by combining the following two maps:

$$
\operatorname{Her}^{+}(2) \ni\left(\begin{array}{cc}
h & \bar{w} \\
w & k
\end{array}\right) \mapsto \frac{1}{2 \sqrt{h k-|w|^{2}}}(h+k, w+\bar{w},-i(w-\bar{w}), h-k) \in L_{1}
$$

and

$$
L_{1} \ni\left(x_{0}, x_{1}, x_{2}, x_{3}\right) \mapsto\left(y_{1}, y_{2}, y_{3}\right)=\frac{1}{1+x_{0}}\left(x_{1}, x_{2}, x_{3}\right) \in \mathbf{B}_{3} .
$$

Through this identification, the image surface is drawn in the ball. The ideal boundary of $\mathbf{H}^{3}$ is naturally identified with the Riemann sphere $\boldsymbol{C} \cup\{\infty\}$. The group of all orientation preserving isometries of $\mathbf{H}^{3}$ is isomorphic to $\mathrm{PGL}_{2}(\boldsymbol{C})$, which acts on the Riemann sphere as well. The hyperbolic Schwarz map $\mathscr{S}$ is singular only along the curve $\{x \in X|| q(x) \mid=1\}$.

In this paper, we study this map for the differential equation with an irregular singularity at infinity:

$$
q(x)=x^{n}\left(q_{0}+q_{1} x^{-1}+q_{2} x^{-2}+\cdots\right), \quad q_{0} \neq 0,
$$

for sufficiently large $x: \quad|x|>R$, and $n=-1,0,1,2, \ldots$ Since solutions of $(E)$ for this $q(x)$ ramify at infinity, $\mathscr{S}$ also ramifies at infinity. Therefore, throughout this paper, we consider $\mathscr{S}$ on the universal covering $\mathscr{R}[\boldsymbol{C} \backslash\{0\}]$ of $\boldsymbol{C} \backslash\{0\}$ 
with $x$ sufficiently large. Let $\theta_{j}(j \in \boldsymbol{Z})$ be the singular directions $(\S 2.2)$. Then we show the following:

- When $x$ goes to infinity along a ray $\{\arg x=\theta\}\left(\theta_{j}<\theta<\theta_{j+1}\right)$, the point $\mathscr{S}(x)$ tends to a boundary point say, $b_{j}$. These $b_{j}$ are mutually related by Stokes coefficients.

- When $x$ goes to infinity along a singular ray $\left\{\arg x=\theta_{j}\right\}$, then $\mathscr{S}(x)$ either accumulates to a circle on the boundary, tends to a boundary point, or accumulates to a circle inside $\mathbf{H}^{3}$.

- When $x$ goes to infinity along a curve $\operatorname{Re} \Lambda(x)=c$ ( $c$ is a constant), where $\Lambda(x)$ is the exponent of the formal solution $(\S 2.1)$, then $\mathscr{S}(x)$ accumulates to a circle on the boundary, and these circles cover the whole sphere, except when $n=0$. (Theorems 3.4 and 3.9.)

The paper is organized as follows. In $\S 2$, we recall how to get formal solutions at infinity, holomorphic solutions asymptotic to them, and Stokes multipliers. In $\S 3$ we get some evaluation of the hyperbolic Schwarz map around the infinity. We also study the cases for $n=-1,0,1$ in a detailed manner. In $\S 4$ we consider the limit points of the image of rays in several sectors under the hyperbolic Schwarz map, and give relations among them in terms of Stokes multipliers.

\section{Formal solutions and asymptotic expansions}

In this section we review some basic facts on the asymptotic expansions of solutions of $(E)$ near an irregular singular point. As a general reference, we refer the reader to $[\mathrm{Si}],[\mathrm{W}]$ and $[\mathrm{T}]$.

\subsection{Construction of formal solutions}

In this subsection we recall how to get formal solutions of $(E)$. These formal solutions play a crucial role in our study. We summarize the result of this subsection as follows:

Proposition 2.1. The equation $(E)$ with (1.1) has two formal solutions $\hat{u}_{+}(x)$ and $\hat{u}_{-}(x)$ that have the form

$$
\hat{u}_{ \pm}(x)=\exp ( \pm \Lambda(x)) x^{-n / 4}\left(1+R_{ \pm}(x)\right)
$$

where

$$
\begin{aligned}
& \Lambda(x)=\lambda_{0} x^{m+1}+\lambda_{1} x^{m}+\cdots+\lambda_{m} x+\alpha \log x, \quad \lambda_{0}=\frac{\sqrt{q_{0}}}{m+1}, \\
& R_{ \pm}(x)=R_{ \pm, 1} x^{-1}+R_{ \pm, 2} x^{-2}+R_{ \pm, 3} x^{-3}+\cdots
\end{aligned}
$$


for $n=2 m$ with non-negative integer $m$, and

$$
\begin{aligned}
& \Lambda(x)=\lambda_{0} x^{m+1 / 2}+\lambda_{1} x^{m-1 / 2}+\cdots+\lambda_{m} x^{1 / 2}, \quad \lambda_{0}=\frac{\sqrt{q_{0}}}{m+1 / 2}, \\
& R_{ \pm}(x)=R_{ \pm, 1} x^{-1 / 2}+R_{ \pm, 2} x^{-1}+R_{ \pm, 3} x^{-3 / 2}+\cdots
\end{aligned}
$$

for $n=2 m-1$. Here $\alpha, \lambda_{l}$ and $R_{ \pm, l}$ are constants.

Let us construct formal solutions of the form (2.1) when $n=2 m$. Suppose that a solution has the form

$$
u(x)=\exp \left(\int^{x} S(x) d x\right) .
$$

Then $S(x)$ should satisfy the Riccati equation

$$
S^{2}+\frac{d S}{d x}=q(x)
$$

associated to $(E)$. Since the degree of the left-hand side of $(2.7)$ with respect to $x$ is $2 m$, we look for a solution of the following form:

$$
S(x)=x^{m}\left(s_{0}+s_{1} x^{-1}+s_{2} x^{-2}+\cdots\right) .
$$

By substituting (2.8) into (2.7) and equating the coefficients of like powers of $x$, we obtain the following recursion relations:

$$
\begin{aligned}
& s_{0}^{2}=q_{0}, \\
& 2 s_{0} s_{k}+\sum_{j=1}^{k-1} s_{j} s_{k-j}-(k-1) s_{k-m-1}=q_{k} \quad(k \geq 1),
\end{aligned}
$$

where $s_{-l}=0(l=1,2,3, \ldots)$. From (2.9) we have

$$
s_{0}= \pm \sqrt{q_{0}},
$$

and we also find that $s_{k}$ for $k \geq 1$ can be determined uniquely and recursively once we fix the sign in (2.11). Thus we have two formal solutions of (2.7) denoted by

$$
S_{ \pm}(x)=x^{m}\left(s_{ \pm, 0}+s_{ \pm, 1} x^{-1}+s_{ \pm, 2} x^{-2}+\cdots\right) .
$$

(These solutions are formal ones because they do not, in general, converge.) By induction, we can see the following relations among these coefficients:

$$
\begin{gathered}
s_{+, j}=-s_{-, j} \quad \text { for } 0 \leq j \leq m, \\
s_{+, m+1}+s_{-, m+1}=-m .
\end{gathered}
$$


The last relation implies

$$
s_{ \pm, m+1}= \pm \alpha-\frac{m}{2}
$$

for some constant $\alpha$. Therefore we obtain

$$
\int^{x} S_{ \pm}(x) d x= \pm \Lambda(x)-\frac{m}{2} \log x+\sum_{j=m+2}^{\infty} \frac{s_{ \pm, j}}{m-j+1} x^{m-j+1}
$$

with

$$
\Lambda(x)=\sum_{j=0}^{m} \frac{s_{+, j}}{m-j+1} x^{m-j+1}+\alpha \log x .
$$

By substituting (2.12) into (2.6), and expanding the terms with negative powers with respect to $x$, we obtain the formal solutions for $n=2 m$.

We can discuss the case where $n=2 m-1$ in a similar manner. In this case we consider the following expansion of $S(x)$ instead of (2.8):

$$
S(x)=x^{m-1 / 2}\left(s_{0}+s_{1 / 2} x^{-1 / 2}+s_{1} x^{-1}+\cdots\right) .
$$

It then follows from the recursion relation of $\left\{s_{j / 2}\right\}$ that

$$
\begin{gathered}
s_{1 / 2}=s_{3 / 2}=\cdots=s_{m-1 / 2}=0, \\
s_{m+1 / 2}=-\frac{1}{2} m+\frac{1}{4} .
\end{gathered}
$$

This gives (2.1) with (2.4). We tabulate examples of formal solutions for $n=-1,0, \ldots, 4$ in Table 1 .

\subsection{Asymptotic expansions of solutions}

The formal solutions constructed in the previous subsection turn out to be the asymptotic expansions of true solutions in some sectors. This is a consequence of a theorem by Poincaré and Hukuhara. Since solutions of $(E)$, in general, ramify at infinity, we study the asymptotic behavior of solutions in the universal covering $\mathscr{R}[\boldsymbol{C} \backslash\{0\}]$ of $\boldsymbol{C} \backslash\{0\}$. We denote by $x=r e^{i \theta}$ a point in $\mathscr{R}[\boldsymbol{C} \backslash\{0\}]$ with positive $r$ and real $\theta$ (we also set $|x|=r, \arg x=\theta$ ).

To give a precise statement of a theorem by Poincaré and Hukuhara, we introduce the notion of singular directions.

The expressions of the formal solutions suggest that the exponential factor $\exp [ \pm \Lambda(x)]$ may be crucial for the asymptotics. The highest degree part of 
Table 1. Some examples of formal solutions.

\begin{tabular}{|c|c|}
\hline$n$ & $q(x)$ and $\hat{u}_{ \pm}(x) \quad\left(q_{0} \neq 0\right)$ \\
\hline-1 & $\begin{aligned} q & =\frac{q_{0}}{x}+\frac{q_{1}}{x^{2}}+\cdots \\
\hat{u}_{ \pm} & =\exp \left[ \pm 2 \sqrt{q_{0}} x^{1 / 2}\right] x^{1 / 4}\left[1+O\left(|x|^{-1 / 2}\right)\right] .\end{aligned}$ \\
\hline 0 & $\begin{aligned} q & =q_{0}+\frac{q_{1}}{x}+\frac{q_{2}}{x^{2}}+\cdots \\
\hat{u}_{ \pm} & =\exp \left[ \pm \sqrt{q_{0}} x\right] x^{ \pm q_{1} /\left(2 \sqrt{q_{0}}\right)}\left(1+O\left(|x|^{-1}\right)\right)\end{aligned}$ \\
\hline 1 & $\begin{aligned} q & =q_{0} x+q_{1}+\frac{q_{2}}{x}+\cdots \\
\hat{u}_{ \pm} & =\exp \left[ \pm\left(\frac{2}{3} \sqrt{q_{0}} x^{3 / 2}+\frac{q_{1}}{\sqrt{q_{0}}} x^{1 / 2}\right)\right] x^{-1 / 4}\left(1+O\left(|x|^{-1 / 2}\right)\right)\end{aligned}$ \\
\hline 2 & $\begin{aligned} q= & q_{0} x^{2}+q_{1} x+q_{2}+\cdots \\
\hat{u}_{ \pm}= & \exp \left[ \pm\left(\frac{\sqrt{q_{0}}}{2} x^{2}+\frac{q_{1}}{2 \sqrt{q_{0}}} x\right)\right] x^{ \pm \alpha-1 / 2}\left(1+O\left(|x|^{-1}\right)\right), \\
& \left(\alpha=\frac{q_{2}}{2 q_{0}^{1 / 2}}-\frac{q_{1}^{2}}{8 q_{0}^{3 / 2}}\right)\end{aligned}$ \\
\hline 3 & $\begin{aligned} q= & q_{0} x^{3}+q_{1} x^{2}+q_{2} x+\cdots \\
\hat{u}_{ \pm}= & \exp \left[ \pm\left(\frac{5}{2} \sqrt{q_{0}} x^{5 / 2}+\frac{q_{1}}{3 \sqrt{q_{0}}} x^{3 / 2}+\left(\frac{q_{2}}{2 \sqrt{q_{0}}}-\frac{q_{1}^{2}}{\sqrt{q_{0}^{3}}}\right) x^{1 / 2}\right)\right. \\
& \times x^{-3 / 4}\left(1+O\left(|x|^{-1 / 2}\right)\right) .\end{aligned}$ \\
\hline 4 & $\begin{aligned} q= & q_{0} x^{4}+q_{1} x^{3}+q_{2} x^{2}+q_{3} x+\cdots, \\
\hat{u}_{ \pm}= & \exp \left[ \pm\left(\frac{\sqrt{q_{0}}}{3} x^{3}+\frac{q_{1}}{4 \sqrt{q_{0}}} x^{2}+\left(\frac{q_{2}}{2 \sqrt{q_{0}}}-\frac{q_{1}^{2}}{8 \sqrt{q_{0}^{3}}}\right) x\right)\right. \\
& \times x^{ \pm \alpha-1}\left(1+O\left(|x|^{-1}\right)\right), \\
& \left(\alpha=\frac{q_{3}}{2 \sqrt{q_{0}}}-\frac{q_{1} q_{2}}{4 \sqrt{q_{0}^{3}}}+\frac{q_{1}^{3}}{16 \sqrt{q_{0}^{5}}}\right)\end{aligned}$ \\
\hline
\end{tabular}

$\Lambda(x)$ is given by

$$
\frac{\sqrt{q_{0}}}{n / 2+1} x^{n / 2+1}=\frac{\sqrt{\left|q_{0}\right|}}{n / 2+1}|x|^{n / 2+1} \exp \left[i\left(\frac{n}{2}+1\right) \theta+\frac{1}{2} i \arg \left(q_{0}\right)\right]
$$

with $x=r e^{i \theta}$. We are thus lead to the definition:

Definition 2.2. The angular value $\theta$ satisfying

$$
\cos \left(\left(\frac{1}{2} n+1\right) \theta+\frac{1}{2} \arg q_{0}\right)=0
$$


is called a singular direction. The ray in $\mathscr{R}[\boldsymbol{C} \backslash\{0\}]$ starting at the origin with the angular value $\theta$ is called a singular ray.

We name the singular direction as

$$
\theta_{j}:=\frac{1}{n / 2+1}\left(\left(j-\frac{1}{2}\right) \pi-\frac{1}{2} \arg q_{0}\right) \quad(j \in \boldsymbol{Z}) .
$$

We call the region in $\mathscr{R}[\boldsymbol{C} \backslash\{0\}]$ of the following form a sector centered at infinity:

$$
\Sigma(\underline{\theta}, \bar{\theta}, R):=\left\{x=r e^{i \theta} ; \underline{\theta}<\theta<\bar{\theta}, r>R\right\} .
$$

Since we consider near the infinity, we always assume that $R$ is sufficiently large. We also call the region of the following form a closed sector:

$$
\bar{\Sigma}(\underline{\theta}, \bar{\theta}, R):=\left\{x=r e^{i \theta} ; \underline{\theta} \leq \theta \leq \bar{\theta}, r \geq R\right\} .
$$

The following special sector $\Sigma_{j}$ near the infinity plays the central role in a theorem by Poincaré and Hukuhara:

$$
\Sigma_{j}:=\Sigma\left(\theta_{j-1}, \theta_{j+1}, R\right)=\left\{x=r e^{i \theta} ; \theta_{j-1}<\theta<\theta_{j+1}, r>R\right\},
$$

where $\theta_{j}$ is a singular direction.

Remark 2.3. The singular rays for $n=-1, \ldots, 4$ in $\{0 \leq \arg x<2 \pi\}$ are illustrated in Figure 1. Since $\theta_{j}$ is congruent to $\theta_{j+n+2}$ modulo $2 \pi$ for any integer $j$, the natural projection of the singular ray for $\theta_{j}$ (resp., $\Sigma_{j}$ ) and that for
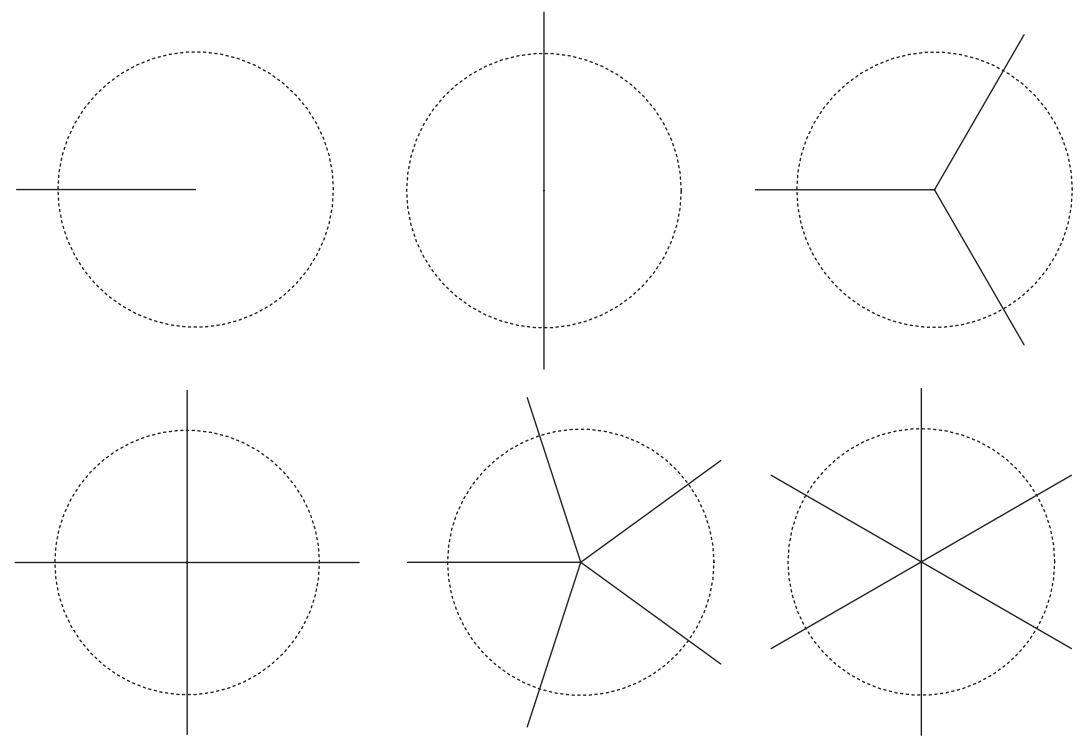

Fig. 1. Singular rays for $n=-1,0,1,2,3,4$ in $\{0 \leq \arg x<2 \pi\}$. 
$\theta_{j+n+2}$ (resp., $\left.\Sigma_{j+n+2}\right)$ to the complex plane are the same. For example, when $n=-1$, all singular rays in the universal covering of $C \backslash\{0\}$ project to the same ray in the complex plane. Note that analytic properties (e.g., asymptotic behavior) of a solution in $\Sigma_{j}$ would be different from those in $\Sigma_{j+n+2}$ since a solution, in general, ramifies at infinity.

In what follows we choose a branch of $\Lambda(x)$ so that the real part of the highest degree part

$$
\frac{\sqrt{q_{0}}}{n / 2+1} x^{n / 2+1}
$$

of $\Lambda(x)$ is positive when $\theta_{0}<\arg x<\theta_{1}$. (Recall that if we fix the highest degree part of $\Lambda(x)$, all terms in $\hat{u}_{+}$or $\hat{u}_{-}$are determined uniquely.) We then analytically continue $\Lambda(x)$ into the universal covering $\mathscr{R}[\boldsymbol{C} \backslash\{0\}]$. By this choice of the branch, the highest degree part of $\operatorname{Re} \Lambda(x)$ is positive (resp., negative) if $\theta_{p}<\arg x<\theta_{p+1}$ with an even $p$ (resp., an odd $p$ ).

Now a theorem by Poincaré and Hukuhara gives

Theorem 2.4. For each $j$, we can find a unique solution $u_{+}^{(j)}\left(\right.$ resp., $\left.u_{-}^{(j)}\right)$ of (E) which can be asymptotically expanded to $\hat{u}_{+}$(resp., $\left.\hat{u}_{+}\right)$in $\Sigma_{j}$; that is, for any closed subsector $\bar{\Sigma} \subset \Sigma_{j}$ we have

$$
u_{+}^{(j)}(x) \sim \hat{u}_{+}(x), \quad u_{-}^{(j)}(x) \sim \hat{u}_{-}(x) \quad(x \rightarrow \infty, x \in \bar{\Sigma}) .
$$

For a proof, we refer to $[\mathrm{T}],[\mathrm{W}]$ and references cited there. For each $j$, a pair $\left(u_{+}^{(j)}, u_{-}^{(j)}\right)$ of solutions becomes a basis of the solution space of $(E)$. In fact, we have the following:

Proposition 2.5. For two solutions $u_{ \pm}^{(j)}$ above, we obtain

$$
W\left[u_{+}^{(j)}, u_{-}^{(j)}\right]=\frac{d u_{+}^{(j)}}{d x} u_{-}^{(j)}-u_{+}^{(j)} \frac{d u_{-}^{(j)}}{d x}=2 \sqrt{q_{0}} .
$$

Proof. We prove this proposition when $n=2 m$; the case $n=2 m-1$ is similarly treated. It follows from the asymptotic expansion of $u_{ \pm}^{(j)}$ and (2.2) that

$$
\begin{aligned}
W\left[u_{+}^{(j)}, u_{-}^{(j)}\right]= & \left(\Lambda^{\prime}(x)-\frac{n}{4 x}\right) x^{-n / 2}\left[1+O\left(|x|^{-1}\right)\right] \\
& -\left(-\Lambda^{\prime}(x)-\frac{n}{4 x}\right) x^{-n / 2}\left[1+O\left(|x|^{-1}\right)\right] \\
= & 2 \Lambda^{\prime}(x) x^{-n / 2}\left[1+O\left(|x|^{-1}\right)\right] \\
= & 2 \sqrt{q_{0}}+O\left(|x|^{-1}\right) .
\end{aligned}
$$

Then, by letting $x \rightarrow \infty$ we have the result, because the Wronskian is a constant. 
Remark 2.6. Although the projections of the sectors $\Sigma_{0}$ and $\Sigma_{n+2}$ to the complex plane are the same (cf. Remark 2.3), $u_{ \pm}^{(0)}$ does not, in general, coincide with $u_{ \pm}^{(n+2)}$. By comparing the asymptotic expansions, we have the following relations:

$$
\left(\begin{array}{l}
u_{+}^{(n+2)}(x) \\
u_{-}^{(n+2)}(x)
\end{array}\right)=e^{-i \pi n / 2}\left(\begin{array}{ll}
0 & 1 \\
1 & 0
\end{array}\right)\left(\begin{array}{l}
u_{+}^{(0)}\left(x e^{-2 i \pi}\right) \\
u_{-}^{(0)}\left(x e^{-2 i \pi}\right)
\end{array}\right) \quad\left(x \in \Sigma_{n+2}\right)
$$

for an odd $n$, and

$$
\left(\begin{array}{l}
u_{+}^{(n+2)}(x) \\
u_{-}^{(n+2)}(x)
\end{array}\right)=e^{-i \pi n / 2}\left(\begin{array}{cc}
e^{2 i \pi \alpha} & 0 \\
0 & e^{-2 i \pi \alpha}
\end{array}\right)\left(\begin{array}{l}
u_{+}^{(0)}\left(x e^{-2 i \pi}\right) \\
u_{-}^{(0)}\left(x e^{-2 i \pi}\right)
\end{array}\right) \quad\left(x \in \Sigma_{n+2}\right)
$$

for an even $n$. Here $u_{ \pm}^{(0)}\left(x e^{-2 i \pi}\right)$ is the analytic continuation of $u_{ \pm}^{(0)}$ along the circle with sufficiently large radius in a clockwise manner. (We use this convention in what follows.)

\subsection{Stokes multipliers}

From Theorem 2.4, we can uniquely determine a pair $\left(u_{+}^{(j)}, u_{-}^{(j)}\right)$ of solutions of $(E)$ which can be asymptotically expanded to $\left(\hat{u}_{+}, \hat{u}_{-}\right)$in $\Sigma_{j}$. Then the following question naturally arises: How $\left(u_{+}^{(j)}, u_{-}^{(j)}\right)$ behaves in the adjacent sector $\Sigma_{j+1}$ of $\Sigma_{j}$.

To study this problem we introduce dominance relations:

Definition 2.7. Let $\Sigma$ be a sector near the infinity. If $\operatorname{Re} \Lambda(x)$ is positive in any closed subsector of $\Sigma$, we say $\hat{u}_{+}$is exponentially large with respect to $\hat{u}_{-}$ in $\Sigma$ and denote it by

$$
\hat{u}_{+} \gg \hat{u}_{-} \quad \text { in } \Sigma \text {. }
$$

If it is negative, we say $\hat{u}_{+}$is exponentially small with respect to $\hat{u}_{-}$and denote it by

$$
\hat{u}_{+} \ll \hat{u}_{-} \quad \text { in } \Sigma \text {. }
$$

Remark 2.8. Because of our choice of the branch of the highest degree part of $\Lambda(x)$ (cf. Remark 2.3), $\hat{u}_{+} \gg \hat{u}_{-}$(resp., $\hat{u}_{+} \ll \hat{u}_{-}$) holds in $\Sigma_{p} \cap \Sigma_{p+1}$, i.e., in a sector $\left\{\theta_{p}<\arg x<\theta_{p+1}\right\}$ with an even $p$ (resp., an odd $p$ ).

Figure 2 illustrates the dominance relations when $p=0$. Since both $\left(u_{+}^{(0)}, u_{-}^{(0)}\right)$ and $\left(u_{+}^{(1)}, u_{-}^{(1)}\right)$ are basis of the solution space of $(E)$ (cf. Proposition 2.5), there exist constants $a, b, c, d$ for which

$$
u_{+}^{(0)}=a u_{+}^{(1)}+b u_{-}^{(1)}, \quad u_{-}^{(0)}=c u_{+}^{(1)}+d u_{-}^{(1)}
$$

hold. Some of these constants can be determined by considering the asymptotic expansions as follows: We consider the first equation of (2.14). It 


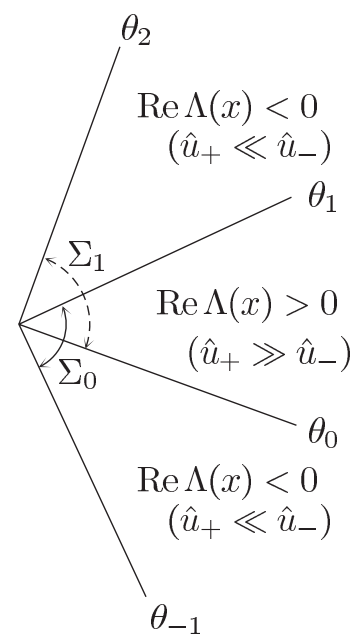

Fig. 2. Sectors and dominance relations in $\Sigma_{0}$ and $\Sigma_{1}$.

follows from the asymptotic expansion of $u_{+}^{(1)}$ in $\Sigma_{0} \cap \Sigma_{1}$, and the dominance relation $u_{+}^{(1)} \gg u_{-}^{(1)}$ in $\Sigma_{0} \cap \Sigma_{1}$ (cf. Remark 2.8) that the asymptotic expansion of the right-hand side of (2.14) is $a \hat{u}_{+}$. On the other hand, that of the left-hand side is $\hat{u}_{+}$; therefore we obtain $a=1$. Next we consider the second equation of (2.14), or

$$
u_{-}^{(0)}-d u_{-}^{(1)}=c u_{+}^{(1)} .
$$

If $c \neq 0$, then the right-hand side of (2.15) is exponentially large in $\Sigma_{0} \cap \Sigma_{1}$ with respect to the left-hand side. Therefore $c$ should be zero. Then, since the asymptotic expansion of the right-hand side is $(1-d) \hat{u}_{-}$, we obtain $d=1$. Thus we obtain $a=d=1$ and $c=0$. After this process the constant $b$ remains undetermined. This constant is called a Stokes multiplier. Though it is generally difficult to determine Stokes multipliers, we give some examples in Appendix A.

This argument leads to

Theorem 2.9. There exist constants $\left\{T_{p}\right\}$ for which

$$
\begin{aligned}
& \left\{\begin{array}{l}
u_{+}^{(p)}=u_{+}^{(p+1)}+T_{p+1} u_{-}^{(p+1)} \\
u_{-}^{(p)}=u_{-}^{(p+1)}
\end{array} \quad \text { (p: even }\right), \\
& \left\{\begin{array}{l}
u_{+}^{(p)}=u_{+}^{(p+1)} \\
u_{-}^{(p)}=u_{-}^{(p+1)}+T_{p+1} u_{+}^{(p+1)}
\end{array} \quad(p: \text { odd })\right.
\end{aligned}
$$

hold in $\Sigma_{p} \cap \Sigma_{p+1}$ for every integer $p$. 


\section{Asymptotic behavior of the hyperbolic Schwarz map}

In this section we fix an integer $p$, and discuss the asymptotic behavior of the hyperbolic Schwarz map $\mathscr{S}$ with $\left(u_{+}^{(p)}, u_{-}^{(p)}\right)$ as a basis, that is, we consider

$$
H^{(p)}=U^{(p) t} \overline{U^{(p)}} \quad \text { with } U^{(p)}=\left(\begin{array}{cc}
u_{+}^{(p)} & u_{+}^{(p)^{\prime}} \\
u_{-}^{(p)} & u_{-}^{(p)^{\prime}}
\end{array}\right) .
$$

Since we fix $p$ in this section, we omit the suffix $p$ throughout this section.

\subsection{Preparation of the estimates of the hyperbolic Schwarz map}

Since we identify $\mathbf{H}^{3}$ with $\mathbf{B}^{3}$, the coordinates of $\mathscr{S}$ are given by

$$
\left(y_{1}, y_{2}, y_{3}\right)=\frac{(2 \operatorname{Re} w, 2 \operatorname{Im} w, h-k)}{h+k+4 \sqrt{\left|q_{0}\right|}},
$$

where

$$
H=\left(\begin{array}{ll}
h & \bar{w} \\
w & k
\end{array}\right)=U(x)^{t} \overline{U(x)} .
$$

We have the expressions

$$
\begin{aligned}
h+k & =\left|u_{+}(x)\right|^{2}+\left|u_{-}(x)\right|^{2}+\left|u_{+}^{\prime}(x)\right|^{2}+\left|u_{-}^{\prime}(x)\right|^{2}, \\
h-k & =\left|u_{+}(x)\right|^{2}-\left|u_{-}(x)\right|^{2}+\left|u_{+}^{\prime}(x)\right|^{2}-\left|u_{-}^{\prime}(x)\right|^{2}, \\
w & =\overline{u_{+}(x)} u_{-}(x)+\overline{u_{+}^{\prime}(x)} u_{-}^{\prime}(x) .
\end{aligned}
$$

We study the asymptotic behavior of $\mathscr{S}$ with the coordinates $\left(y_{1}, y_{2}, y_{3}\right)$. In what follows, we set $r=|x|$. As a preparation for it we prove

Lemma 3.1. For an even $n$,

$$
\begin{aligned}
h= & \left(1+\left|\Lambda^{\prime}(x)-\frac{n}{4 x}\right|^{2}\right) e^{2 \operatorname{Re} \Lambda(x)} r^{-n / 2}\left[1+O\left(r^{-1}\right)\right], \\
k= & \left(1+\left|\Lambda^{\prime}(x)+\frac{n}{4 x}\right|^{2}\right) e^{-2 \operatorname{Re} \Lambda(x)} r^{-n / 2}\left[1+O\left(r^{-1}\right)\right], \\
w= & \left(-\left|\Lambda^{\prime}(x)\right|^{2}+i \frac{n}{2 r} \operatorname{Im}\left(\Lambda^{\prime}(x) e^{i \theta}\right)+1+\frac{n^{2}}{4 r^{2}}\right) \\
& \times e^{-2 i \operatorname{Im} \Lambda(x)} r^{-n / 2}\left[1+O\left(r^{-1}\right)\right] .
\end{aligned}
$$

For an odd $n$, the above relations hold if we replace $O\left(r^{-1}\right)$ with $O\left(r^{-1 / 2}\right)$. 
Proof. We give a proof for an even $n$ (the odd $n$ case is similarly proved). Since $\hat{u}_{ \pm}(x)$ are given by (2.1), and the derivatives of $u_{ \pm}(x)$ are estimated as

$$
u_{ \pm}^{\prime}(x)=\left( \pm \Lambda^{\prime}(x)-\frac{n}{4 x}\right) x^{-n / 4} e^{ \pm \Lambda(x)}\left[1+O\left(|x|^{-1}\right)\right],
$$

we obtain the following:

$$
\begin{aligned}
\left|u_{ \pm}(x)\right|^{2}= & e^{ \pm 2 \operatorname{Re} \Lambda(x)} r^{-n / 2}\left[1+O\left(r^{-1}\right)\right], \\
\left|u_{ \pm}^{\prime}(x)\right|^{2}= & \left| \pm \Lambda^{\prime}(x)-\frac{n}{4 x}\right|^{2} e^{ \pm 2 \operatorname{Re} \Lambda(x)} r^{-n / 2}\left[1+O\left(r^{-1}\right)\right], \\
\overline{u_{+}(x)} u_{-}(x)= & e^{\overline{\Lambda(x)}-\Lambda(x)} r^{-n / 2}\left[1+O\left(r^{-1}\right)\right], \\
\overline{u_{+}^{\prime}(x)} u_{-}^{\prime}(x)= & \overline{\left(\Lambda^{\prime}(x)-\frac{n}{4 x}\right)}\left(-\Lambda^{\prime}(x)-\frac{n}{4 x}\right) \\
& \times e^{\overline{\Lambda(x)}-\Lambda(x)} r^{-n / 2}\left[1+O\left(r^{-1}\right)\right] .
\end{aligned}
$$

Then (3.3)-(3.5) are direct consequences of the above.

\subsection{A detailed estimate for the case $n=0$}

In this subsection we treat the differential equation with

$$
q(x)=\kappa^{2}+\frac{2 a}{x}+\cdots \quad(\kappa \neq 0)
$$

in a detailed manner. The singular directions are

$$
\theta_{j}=j \pi-\frac{\pi}{2}-\arg \kappa
$$

and the corresponding sectors are

$$
\Sigma_{j}=\left\{x=r e^{i \theta} ; r>R,\left|\theta-\theta_{j}\right|<\pi\right\} .
$$

The related data are

$$
\Lambda(x)=\kappa x+\frac{a}{\kappa} \log x,
$$

and the asymptotic expansions

$$
u_{ \pm}(x) \sim e^{ \pm \Lambda(x)}\left[1+O\left(|x|^{-1}\right)\right]=e^{ \pm \kappa x} x^{ \pm a / \kappa}\left[1+O\left(|x|^{-1}\right)\right] .
$$

Hence we obtain

$$
\left|\Lambda^{\prime}(x) \pm \frac{n}{4 x}\right|^{2}=\left|\kappa+\frac{a}{\kappa x}\right|^{2}=|\kappa|^{2}+O\left(r^{-1}\right),
$$


and

$$
\begin{aligned}
h+k & =\left(e^{2 \operatorname{Re} \Lambda(x)}+e^{-2 \operatorname{Re} \Lambda(x)}\right)\left[1+|\kappa|^{2}+O\left(r^{-1}\right)\right], \\
h-k & =\left(e^{2 \operatorname{Re} \Lambda(x)}-e^{-2 \operatorname{Re} \Lambda(x)}\right)\left[1+|\kappa|^{2}+O\left(r^{-1}\right)\right], \\
w & =e^{-2 i \operatorname{Im} \Lambda(x)}\left[1-|\kappa|^{2}+O\left(r^{-1}\right)\right] .
\end{aligned}
$$

We start our argument with recalling the results given in $[\mathrm{SY}, \S 5]$. In [SY] the asymptotic behavior of $\mathscr{S}$ along the ray $\arg x=\dot{\theta}$, where $\dot{\theta}$ is a constant, was studied. For the sake of simplicity we assume that $a$ is real and $\kappa$ is real positive for a while. Since

$$
\operatorname{Re} \Lambda\left(r e^{i \grave{\theta}}\right)=r \kappa \cos \stackrel{\circ}{ }+\frac{a}{\kappa} \log r
$$

holds along the ray $x=r e^{i \stackrel{\theta}{\theta}}$ with $r>0$, we obtain

$$
\begin{array}{ll}
\operatorname{Re} \Lambda\left(r e^{i \theta}\right) \rightarrow+\infty & \text { if } \cos \stackrel{\circ}{\theta}>0, \\
\operatorname{Re} \Lambda\left(r e^{i \ell}\right) \rightarrow-\infty & \text { if } \cos \stackrel{\circ}{\theta}<0,
\end{array}
$$

when $r$ tends to infinity. Hence the hyperbolic Schwarz image of the ray tends to $(0,0,1)$ or $(0,0,-1)$ respectively (cf. (3.2)). If $\cos \ddot{\theta}=0$, i.e, if $\dot{\theta}$ is a singular direction $\theta_{2 j-1}(j \in \boldsymbol{Z})$, the behavior of the hyperbolic Schwarz image depends on the signature of $a / \kappa$. Since

$$
\begin{array}{ll}
\operatorname{Re} \Lambda\left(r e^{i \theta}\right) \rightarrow+\infty & \text { if } a>0, \\
\operatorname{Re} \Lambda\left(r e^{i \ell}\right) \rightarrow-\infty & \text { if } a<0
\end{array}
$$

hold when $\cos \stackrel{\circ}{=} 0$, we find that the image tends to $(0,0,1)$ or $(0,0,-1)$ respectively as $r$ tends to infinity.

If $\stackrel{\circ}{\theta}$ is a singular direction and $a=0$, the image of the ray $\{\arg x=\stackrel{\circ}{\theta}\}$ under $\mathscr{S}$ does not converge to a point, but accumulates to the circle as $|x|$ tends to infinity. In fact it follows from (3.2), (3.3), (3.4) and (3.5) that the image behaves like

$$
\left\{\begin{array}{l}
y_{1}=\frac{\left(1-|\kappa|^{2}\right) \cos (2 \operatorname{Im} \Lambda)}{(1+|\kappa|)^{2}}+O\left(r^{-1}\right), \\
y_{2}=-\frac{\left(1-|\kappa|^{2}\right) \sin (2 \operatorname{Im} \Lambda)}{(1+|\kappa|)^{2}}+O\left(r^{-1}\right), \\
y_{3}=O\left(r^{-1}\right) .
\end{array}\right.
$$


Thus we conclude that the image of the ray under $\mathscr{S}$ accumulates to

$$
\left\{y_{1}^{2}+y_{2}^{2}=\left(\frac{1-|\kappa|^{2}}{(1+|\kappa|)^{2}}\right)^{2}, y_{3}=0\right\} \text {. }
$$

We can also study the case when $\kappa$ and $a$ are complex numbers in a similar manner. The result is summarized as

Proposition 3.2 (Cf. [SY, Proposition 5.1]). Let the coefficient $q(x)$ of the equation is given as (3.6).

(1) If $\theta_{p}<\stackrel{\circ}{\theta} \theta_{p+1}$, then the image of the ray $x=r e^{i \ominus}$ under the hyperbolic Schwarz map tends to $(0,0,1)$ if $p$ is even, and tends to $(0,0,-1)$ if $p$ is odd as $r$ tends to infinity.

(2) If $\dot{\theta}$ is a singular direction, then the image of the ray $x=r e^{i \theta}$ under the hyperbolic Schwarz map tends to $(0,0,1)$ if $\operatorname{Re}(a / \kappa)>0$, tends to $(0,0,-1)$ if $\operatorname{Re}(a / \kappa)<0$, and accumulates to the circle

$$
C:\left\{\begin{array}{l}
y_{1}^{2}+y_{2}^{2}=\left(\frac{2\left(1-|\kappa|^{2}\right)}{\left(e^{2 c}+e^{-2 c}\right)\left(1+|\kappa|^{2}\right)+4|\kappa|}\right)^{2}, \\
y_{3}=\frac{\left(1+|\kappa|^{2}\right)\left(e^{2 c}-e^{-2 c}\right)}{\left(1+|\kappa|^{2}\right)\left(e^{2 c}+e^{-2 c}\right)+4|\kappa|},
\end{array}\right.
$$

if $\operatorname{Re}(a / \kappa)=0$, where

$$
c:=\operatorname{Re} \Lambda\left(r e^{i \dot{\theta}}\right)=-\operatorname{Im}\left(\frac{a}{\kappa}\right) \stackrel{\circ}{ } .
$$

Remark 3.3. More generally, consider the curve $x(t)=r(t) e^{i \theta(t)}$, where $r(t)$ is a positive function which tends to infinity as $t$ tends to infinity, and $\theta(t)$ satisfies either

$$
\text { (i) }-\frac{\pi}{2}+\varepsilon \leq \theta(t) \leq \frac{\pi}{2}-\varepsilon \quad \text { or } \quad \text { (ii) } \quad \frac{\pi}{2}+\varepsilon \leq \theta(t) \leq \frac{3 \pi}{2}-\varepsilon
$$

for a positive constant $\varepsilon$. Then there exists a positive $\delta$ for which

$$
\text { (i) } \cos \theta(t) \geq \delta \quad \text { or } \quad \text { (ii) } \cos \theta(t) \leq-\delta
$$

holds, and we can apply the same argument with the case that $\theta(t)$ is a constant to conclude that the hyperbolic Schwarz image of the ray tends to $(0,0,1)$ or $(0,0,-1)$.

Thus the hyperbolic Schwarz image tends along a ray to some point if we choose the parameter $a$ generically and, interestingly, it accumulates to the circle $C$ for some specially chosen parameter. As we see below, such an accumu- 

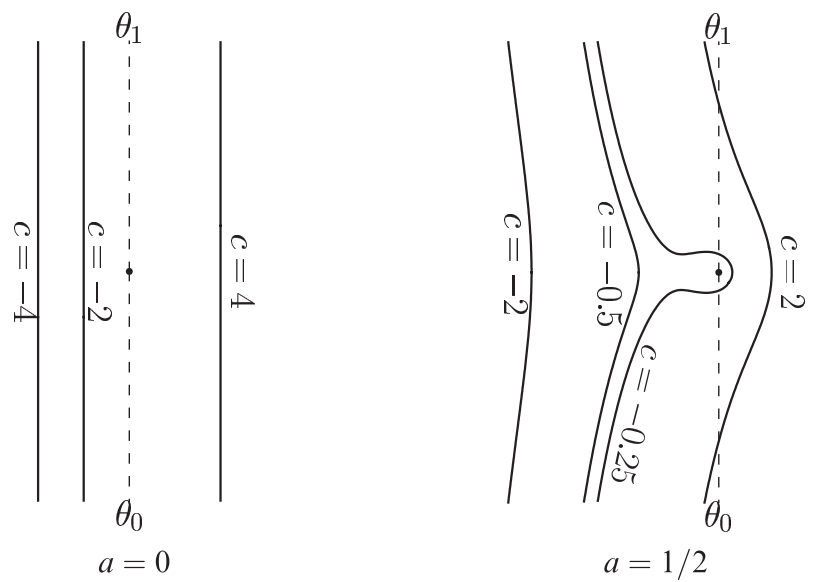

Fig. 3. Examples of the curve $\operatorname{Re} \Lambda(x)=c$ for $q(x)=1 / 2+2 a x^{-1}+\cdots$. Here dotted lines indicate singular rays.

lation to a circle occurs for any parameter $a$ if we appropriately choose a curve instead of a ray.

From the estimates (3.3) through (3.5), we find that the asymptotic behavior of the hyperbolic Schwarz map is governed by $\operatorname{Re} \Lambda(x)$. Therefore we consider the curve

$$
\operatorname{Re} \Lambda(x)=\operatorname{Re}\left[\kappa x+\frac{a}{\kappa} \log x\right]=c
$$

with any real constant $c$. Let $x$ be on the curve $\operatorname{Re} \Lambda(x)=c$ (cf. Figure 3 for examples of this curve); then, it follows from (3.7), (3.8), (3.9) and (3.2) that the coordinates $\left(y_{1}, y_{2}, y_{3}\right)$ behave as

$$
\begin{aligned}
& y_{1}=\frac{2\left(1-|\kappa|^{2}\right) \cos (2 \operatorname{Im} \Lambda(x))}{\left(1+|\kappa|^{2}\right)\left(e^{2 c}+e^{-2 c}\right)+4|\kappa|}+O\left(r^{-1}\right), \\
& y_{2}=\frac{-2\left(1-|\kappa|^{2}\right) \sin (2 \operatorname{Im} \Lambda(x))}{\left(1+|\kappa|^{2}\right)\left(e^{2 c}+e^{-2 c}\right)+4|\kappa|}+O\left(r^{-1}\right), \\
& y_{3}=\frac{\left(1+|\kappa|^{2}\right)\left(e^{2 c}-e^{-2 c}\right)}{\left(1+|\kappa|^{2}\right)\left(e^{2 c}+e^{-2 c}\right)+4|\kappa|}+O\left(r^{-1}\right) .
\end{aligned}
$$

Since $|\operatorname{Im} \Lambda(x)|$ monotonically increases along the curve $\operatorname{Re} \Lambda(x)=c \quad$ (cf. Lemma 3.7), we obtain

Theorem 3.4. Let the coefficient $q$ of the equation be given as (3.6). 

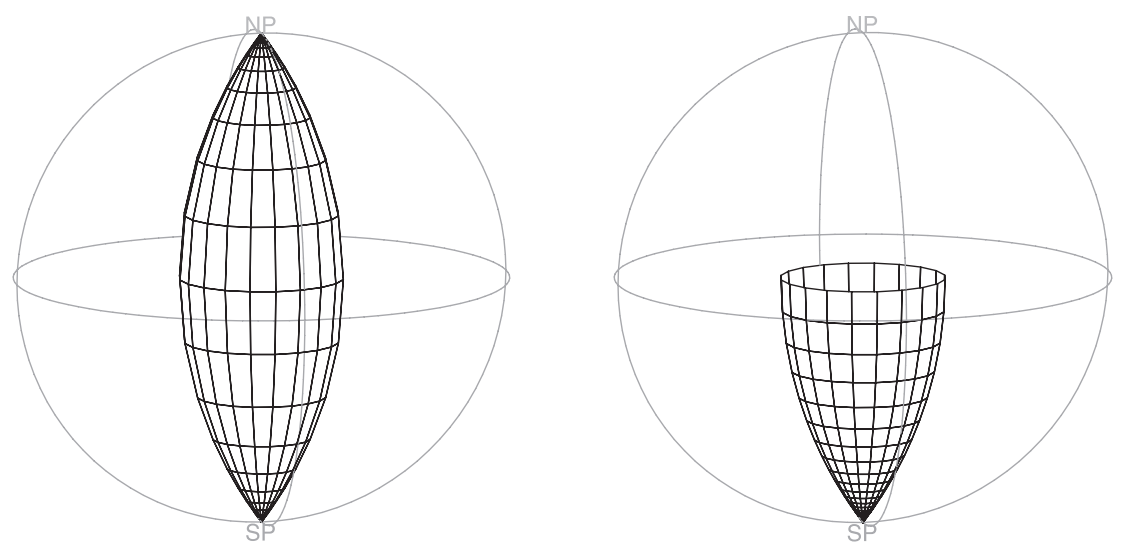

Fig. 4. Hyperbolic cylinder.

(1) The image of the curve $\operatorname{Re} \Lambda(x)=c$ under the hyperbolic Schwarz map tends to the circle

$$
\begin{aligned}
y_{1}^{2}+y_{2}^{2} & =\left(\frac{2\left(1-|\kappa|^{2}\right)}{\left(1+|\kappa|^{2}\right)\left(e^{2 c}+e^{-2 c}\right)+4|\kappa|}\right)^{2}, \\
y_{3} & =\frac{\left(1+|\kappa|^{2}\right)\left(e^{2 c}-e^{-2 c}\right)}{\left(1+|\kappa|^{2}\right)\left(e^{2 c}+e^{-2 c}\right)+4|\kappa|} .
\end{aligned}
$$

(2) As c tends to $\infty$ or $-\infty$, the circle shrinks to the north pole $(0,0,1)$ or to the south pole $(0,0,-1)$, respectively.

(3) The circles for $-\infty<c<\infty$ make a surface, called a hyperbolic cylinder.

Remark 3.5. A hyperbolic cylinder is one of closed flat surfaces in the hyperbolic 3-space and it looks like the surface in Figure 4.

\subsection{The case $n=1$, a generalization of Airy differential equation}

We consider the case $n=1$; set

$$
q(x)=x+q_{1}+q_{2} x^{-1}+\cdots .
$$

A special case $q=x$ corresponds to the Airy differential equation, which is studied in $[\mathrm{SY}]$. The singular directions for this $q(x)$ are

$$
\theta_{j}=\frac{2}{3} j \pi-\frac{\pi}{3}
$$


and the corresponding sectors are

$$
\Sigma_{j}=\left\{x=r e^{i \theta} ; r>R,\left|\theta-\theta_{j}\right|<\frac{2}{3} \pi\right\} .
$$

For each sector, let $u_{ \pm}$denote the solutions with the prescribed asymptotic expansions:

$$
u_{ \pm}=e^{ \pm \Lambda(x)} x^{1 / 4}\left[1+O\left(|x|^{-1 / 2}\right)\right] \quad \text { with } \Lambda(x)=\frac{2}{3} x^{3 / 2}+q_{1} x^{1 / 2} .
$$

In this case,

$$
\left|\Lambda^{\prime}(x)-\frac{1}{4 x}\right|^{2}=\left|x^{1 / 2}+\frac{1}{2} q_{1} x^{-1 / 2}-\frac{1}{4} x^{-1}\right|^{2}=r\left[1+O\left(r^{-1}\right)\right]
$$

and

$$
\begin{aligned}
h+k & =e^{2 \operatorname{Re} \Lambda(x)} r^{1 / 2}\left[1+O\left(r^{-1 / 2}\right)\right]+e^{-2 \operatorname{Re} \Lambda(x)} r^{1 / 2}\left[1+O\left(r^{-1 / 2}\right)\right], \\
h-k & =e^{2 \operatorname{Re} \Lambda(x)} r^{1 / 2}\left[1+O\left(r^{-1 / 2}\right)\right]-e^{-2 \operatorname{Re} \Lambda(x)} r^{1 / 2}\left[1+O\left(r^{-1 / 2}\right)\right], \\
w & =-e^{-2 i \operatorname{Im} \Lambda(x)} r^{1 / 2}\left[1+O\left(r^{-1 / 2}\right)\right] .
\end{aligned}
$$

As in the case $n=0$, we can confirm that the image of a ray $x(t)=t e^{i \dot{\theta}}$ with $\stackrel{\circ}{p}_{p}<\theta<\dot{\theta}_{p+1}$, where $p$ is an even (resp., odd) integer, tends to $(0,0,1)$ (resp., $(0,0,-1))$ as $\theta \rightarrow \infty$.

The asymptotic behavior along the curve $\operatorname{Re} \Lambda(x)=c$ (cf. Figure 5) as $x \rightarrow \infty$ is given by

$$
\begin{aligned}
h+k & =r^{1 / 2}\left[e^{2 c}+e^{-2 c}+O\left(r^{-1 / 2}\right)\right], \\
h-k & =r^{1 / 2}\left[e^{2 c}-e^{-2 c}+O\left(r^{-1 / 2}\right)\right], \\
w & =-e^{-2 i \operatorname{Im} \Lambda(x)} r^{1 / 2}\left[1+O\left(r^{-1 / 2}\right)\right]
\end{aligned}
$$

and

$$
\begin{aligned}
& y_{1}=\frac{2 \operatorname{Re} w}{h+k+4}=-\frac{2 \cos [2 \operatorname{Im} \Lambda(x)]}{e^{2 c}+e^{-2 c}}+O\left(r^{-1 / 2}\right), \\
& y_{2}=\frac{2 \operatorname{Im} w}{h+k+4}=\frac{2 \sin [2 \operatorname{Im} \Lambda(x)]}{e^{2 c}+e^{-2 c}}+O\left(r^{-1 / 2}\right), \\
& y_{3}=\frac{h-k}{h+k+4}=\frac{e^{2 c}-e^{-2 c}}{e^{2 c}+e^{-2 c}}+O\left(r^{-1 / 2}\right) .
\end{aligned}
$$




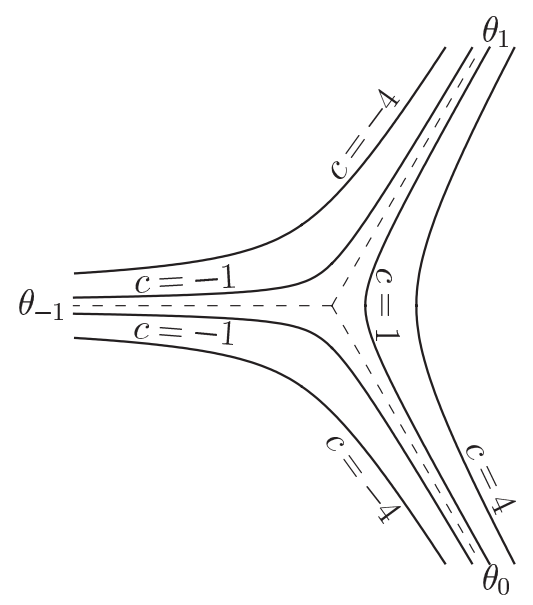

$q_{1}=0$

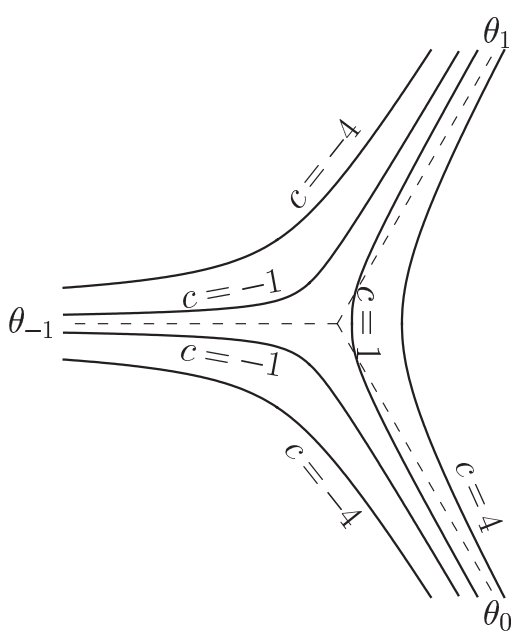

$q_{1}=1$

Fig. 5. Examples of the curve $\operatorname{Re} \Lambda(x)=c$ for $q(x)=x+q_{1}+\cdots$. Here dotted lines indicate singular rays.

Therefore, the image points approach to the circle

$$
y_{1}^{2}+y_{2}^{2}=\left(\frac{2}{e^{2 c}+e^{-2 c}}\right)^{2}, \quad y_{3}=\frac{e^{2 c}-e^{-2 c}}{e^{2 c}+e^{-2 c}},
$$

which lies on the boundary sphere of $\mathbf{B}^{3}$.

\subsection{The case $n=-1$}

The relevant $q$ is

$$
q(x)=\frac{1}{4 x}+\frac{q_{1}}{x^{2}}+\cdots
$$

The singular directions $\theta_{j}$ and sectors $\Sigma_{j}$ are given respectively by

$$
\theta_{j}=2 j \pi-\pi, \quad \Sigma_{j}=\left\{x=r e^{i \theta} ; r>R,\left|\theta-\theta_{j}\right|<2 \pi\right\},
$$

and the asymptotic expansions are

$$
u_{ \pm}(x)=e^{ \pm x^{1 / 2}} x^{1 / 4}\left[1+O\left(|x|^{-1 / 2}\right)\right] .
$$

Since $\Lambda(x)=\lambda(x)=x^{1 / 2}$, we have

$$
\left|\Lambda^{\prime}(x)+\frac{1}{4 x}\right|^{2}=\left|x^{-1 / 2}+\frac{1}{4 x}\right|^{2}=\frac{1}{16 r}\left[1+O\left(r^{-1 / 2}\right)\right]=O\left(r^{-1}\right) .
$$




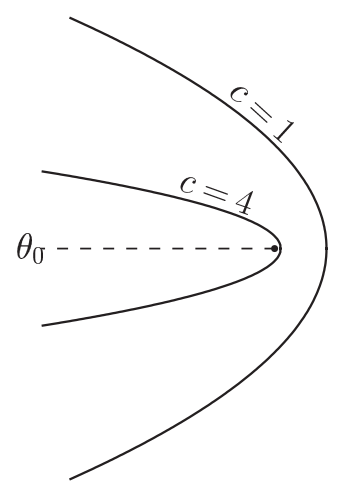

Fig. 6. Examples of the curve $\operatorname{Re} \Lambda(x)=c$ for $q(x)=1 / x+\cdots$. Here dotted lines indicate singular rays.

The same reasoning as before shows that the image of the curve $\operatorname{Re} \Lambda(x)=c$ (cf. Figure 6) is expressed as

$$
\begin{aligned}
& y_{1}=\frac{2 \operatorname{Re} w}{h+k+1}=\frac{2 \cos (2 \operatorname{Im} \Lambda(x))}{e^{2 c}+e^{-2 c}}+O\left(r^{-1 / 2}\right), \\
& y_{2}=\frac{2 \operatorname{Im} w}{h+k+1}=\frac{-2 \sin (2 \operatorname{Im} \Lambda(x))}{e^{2 c}+e^{-2 c}}+O\left(r^{-1 / 2}\right), \\
& y_{3}=\frac{h-k}{h+k+1}=\frac{e^{2 c}-e^{-2 c}}{e^{2 c}+e^{-2 c}}+O\left(r^{-1 / 2}\right),
\end{aligned}
$$

which implies that it accumulates also to the circle (3.12). Along any curve $x(t)$ for which $\operatorname{Re} \Lambda(x(t)) \rightarrow \pm \infty$, the image tends to $(0,0, \pm 1)$.

\subsection{The curve $\operatorname{Re} \Lambda(x)=c$}

For the study of asymptotic behavior of the hyperbolic Schwarz map, the formulas in the previous subsection suggest that the curve defined by the equation $\operatorname{Re} \Lambda(x)=c$, where $c$ is a constant, is essential for general $n$. In this subsection we study its asymptotic behavior. (See Figures 3, 5 and 6 for such examples. See also Figure 7 for $q(x)=x^{2}+q_{1} x+q_{2}+\cdots$.)

Generally the curve $\operatorname{Re} \Lambda(x)=c$ is different from singular lines. For example, consider the case $\Lambda(x)=x^{2}+x$. The singular directions are $\pi / 4$, $3 \pi / 4,5 \pi / 4,7 \pi / 4$, etc., and, for $x=\xi+i \eta$, the singular lines are $\xi= \pm \eta$. Since $\Lambda(x)=\xi^{2}-\eta^{2}+\xi+2 i \xi \eta$, the curve $\operatorname{Re} \Lambda(x)=c$ is written as $\eta^{2}=\xi^{2}+\xi-c$ and each component of the curve tends to one of the lines

$$
\eta= \pm\left(\xi+\frac{1}{2}\right)
$$



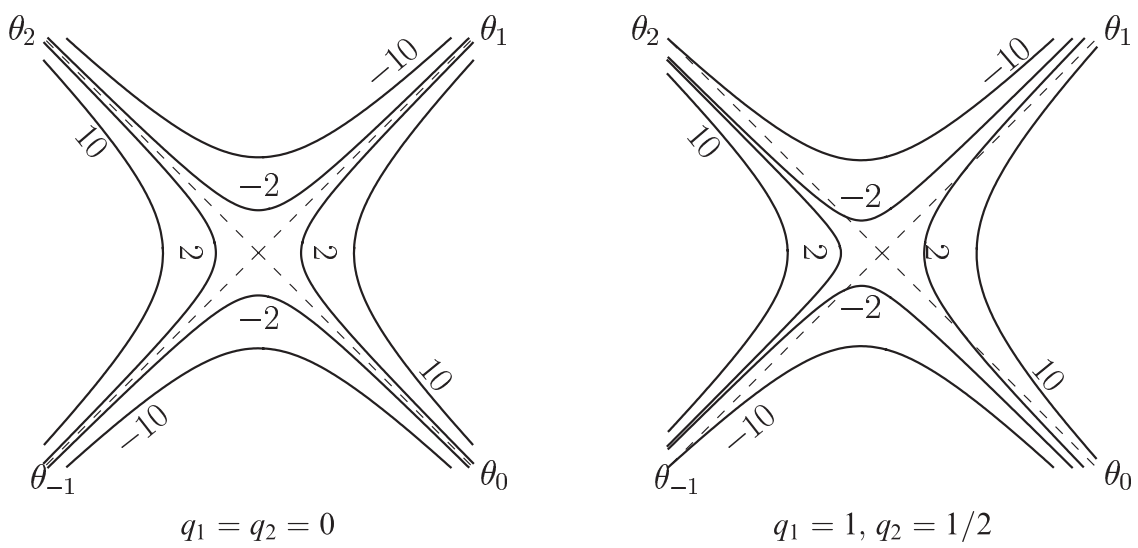

Fig. 7. Examples of the curve $\operatorname{Re} \Lambda(x)=c$ for $q(x)=x^{2}+q_{1} x+q_{2}+\cdots$. Here dotted lines indicate singular rays, and a number attached to a curve is the value of $c$.

as $|x| \rightarrow \infty$; hence, the tangent to each curve tends to a singular direction. This description is valid generally, because

$$
\begin{aligned}
\operatorname{Re} \Lambda\left(r e^{i \theta}\right)= & \frac{\sqrt{\left|q_{0}\right|}}{(n / 2)+1} r^{(n / 2)+1} \cos \left(\left(\frac{n}{2}+1\right) \theta+\frac{1}{2} \arg q_{0}\right) \\
& +\left|\lambda_{1}\right| r^{n / 2} \cos \left(\frac{n}{2} \theta+\arg \lambda_{1}\right)+\cdots
\end{aligned}
$$

implies that $\theta$ such that $\operatorname{Re} \Lambda\left(r e^{i \theta}\right)=c$ tends to a singular direction as $r \rightarrow \infty$.

We define a vector field $L$ by

$$
L=i \frac{\overline{d \Lambda}}{d x}=\left(-\frac{\partial}{\partial \eta} \operatorname{Re} \Lambda(x), \frac{\partial}{\partial \xi} \operatorname{Re} \Lambda(x)\right) .
$$

Since

$$
\frac{d}{d t} \operatorname{Re} \Lambda(x(t))=\operatorname{Re}\left(\frac{d}{d t} \Lambda(x(t))\right)=\operatorname{Re}\left(\frac{d \Lambda}{d x} \cdot \dot{x}(t)\right),
$$

for any curve $x(t)$, we have the following:

Lemma 3.6. The vector field $L$ is parallel to the tangent vector of the curve $\operatorname{Re} \Lambda(x)=c . \quad$ It is singular at the origin (due to the term $\log x$ in $\Lambda(x)$ ) and the zeros of $d \Lambda / d x$.

The curve $\operatorname{Re} \Lambda(x)=c$ is an integral curve of the equation

$$
\dot{x}(t)=i \frac{\overline{d \Lambda}}{d x}
$$

with an initial condition $\operatorname{Re} \Lambda\left(x\left(t_{0}\right)\right)=c$. Moreover, we have 
Lemma 3.7. Let $x(t)$ be the integral curve given above. If it does not pass through any singular point of the vector field, then $\operatorname{Im} \Lambda(x(t))$ increases as $t$ tends to $\infty$ and decreases as $t$ tends to $-\infty$.

Proof. By the assumption, the value

$$
\frac{d}{d t} \operatorname{Im} \Lambda(x(t))=\operatorname{Im}\left(\frac{d \Lambda}{d x} \cdot \dot{x}(t)\right)=\operatorname{Im}\left(i\left|\frac{d \Lambda}{d x}\right|^{2}\right)=\left|\frac{d \Lambda}{d x}\right|^{2}
$$

is always positive.

\subsection{The case $n \geq 1$}

We treat finally the case $n \geq 1$, which includes the case $n=1$ in Subsection 3.3. Since the reasoning is very similar to that case, we only give a sketch. We have $\Lambda^{\prime}(x)=\sqrt{q_{0}} x^{n / 2}+\cdots$ and it implies

$$
1+\left|\Lambda^{\prime}(x) \pm \frac{n}{4 x}\right|^{2}=\left|q_{0}\right| r^{n}\left[1+O\left(r^{-1}\right)\right] .
$$

Hence we obtain

$$
\begin{aligned}
h+k & =\left|q_{0}\right| e^{2 \operatorname{Re} \Lambda(x)} r^{n / 2}\left[1+O\left(r^{-1}\right)\right]+\left|q_{0}\right| e^{-2 \operatorname{Re} \Lambda(x)} r^{n / 2}\left[1+O\left(r^{-1}\right)\right], \\
h-k & =\left|q_{0}\right| e^{2 \operatorname{Re} \Lambda(x)} r^{n / 2}\left[1+O\left(r^{-1}\right)\right]-\left|q_{0}\right| e^{-2 \operatorname{Re} \Lambda(x)} r^{n / 2}\left[1+O\left(r^{-1}\right)\right], \\
w & =-\left|q_{0}\right| e^{-2 i \operatorname{Im} \Lambda(x)} r^{n / 2}\left[1+O\left(r^{-1}\right)\right] .
\end{aligned}
$$

Along any curve $x(t)$ for which $\operatorname{Re} \Lambda(x(t)) \rightarrow \pm \infty$, the image point tends

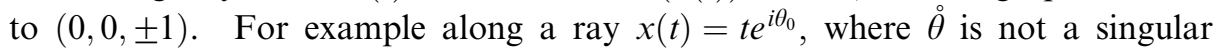
direction, $\left(y_{1}, y_{2}, y_{3}\right)$ tends to $(0,0, \pm 1)$.

Remark 3.8. If $\dot{\theta}$ is one of singular directions, the behavior at infinity depends on the lower order term of $\Lambda(x)$, which we omit here to describe.

On the other hand, along the curve defined by $\operatorname{Re} \Lambda(x)=c$, the coordinates behave as

$$
\begin{aligned}
& y_{1}=\frac{2 \operatorname{Re} w}{h+k+4 \sqrt{\left|q_{0}\right|}}=\frac{-2 \cos (2 \operatorname{Im} \Lambda(x))}{e^{2 c}+e^{-2 c}}+O\left(r^{-1}\right), \\
& y_{2}=\frac{2 \operatorname{Im} w}{h+k+4 \sqrt{\left|q_{0}\right|}}=\frac{2 \sin (2 \operatorname{Im} \Lambda(x))}{e^{2 c}+e^{-2 c}}+O\left(r^{-1}\right), \\
& y_{3}=\frac{h-k}{h+k+4 \sqrt{\left|q_{0}\right|}}=\frac{e^{2 c}-e^{-2 c}}{e^{2 c}+e^{-2 c}}+O\left(r^{-1}\right) .
\end{aligned}
$$

As $x \rightarrow \infty$ along the curve, the image points accumulate to the circle (3.12) (cf. Lemma 3.7). 


\subsection{Summary}

Summarizing the computations in the previous subsections, we have

Theorem 3.9. Assume $n=-1$ or $n \geq 1$.

(I) The image of the ray $\{\arg x=\stackrel{\circ}{\theta}\}$, where $\stackrel{\circ}{\theta}$ is not a singular direction, under the hyperbolic Schwarz map $\mathscr{S}$ tends to the north pole $(0,0,1)$ or to the south pole $(0,0,-1)$ as $x$ tends to infinity along a ray $\{\arg x=\ddot{\theta}\}$. The former case occurs if $\operatorname{Re} \Lambda(x) \rightarrow \infty$ along the ray, and the latter case occurs if $\operatorname{Re} \Lambda(x) \rightarrow-\infty$.

(II) (1) Along the curve $\operatorname{Re} \Lambda(x)=c$, the image $\left(y_{1}, y_{2}, y_{3}\right)$ of the hyperbolic Schwarz map tends to the circle

$$
y_{1}^{2}+y_{2}^{2}=\left(\frac{2}{e^{2 c}+e^{-2 c}}\right)^{2}, \quad y_{3}=\frac{e^{2 c}-e^{-2 c}}{e^{2 c}+e^{-2 c}},
$$

which lies on the boundary sphere.

(2) As c tends to $\infty$ or $-\infty$, the circle shrinks to the north pole $(0,0,1)$ or to the south pole $(0,0,-1)$, respectively.

(3) The union of the circles for all c covers the boundary sphere.

\section{Analytic continuation of hyperbolic Schwarz map}

As we saw in the previous section, the limit point of the image of a ray under the hyperbolic Schwarz map $\mathscr{S}$ drastically changes if we cross a singular ray of $(E)$. To make our argument specific, we consider the image of $\mathscr{S}$ in $\Sigma_{1}$, and chose a pair $\left(u_{+}^{(1)}, u_{-}^{(1)}\right)$ as a basis of $(E)$. If a ray $\{\arg x=\stackrel{\circ}{\theta}\}$ is in $\Sigma_{0} \cap \Sigma_{1}$ (i.e., $\left.\theta_{0}<\stackrel{\circ}{0}<\theta_{1}\right)$, then $\operatorname{Re} \Lambda(x) \rightarrow+\infty$, and hence the image of the ray under $\mathscr{S}$ tends to the north pole $(0,0,1)$ (cf. Theorem 3.9). Now we rotate the ray

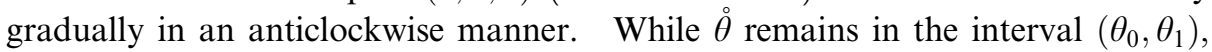
such a limit point never change; the image of the ray approaches to the north pole. If $\dot{\theta}$ goes beyond $\theta_{1}$, however, the image of the ray now approaches to the south pole $(0,0,-1)$. This is a kind of Stokes phenomena. Such a phenomenon of $\mathscr{S}$ was firstly observed in [SY] for the Airy equation and the confluent hypergeometric function, and we have discussed it in a more general situation in the previous section.

In this section we see what occurs if $\dot{\theta}$ goes beyond $\theta_{2}$. Note that our argument in the previous section is done only in the fixed sectors. Therefore to determine the limit point of the image of the ray for $\stackrel{\theta}{>} \theta_{2}$, we must consider the analytic continuation of solutions of $(E)$ (and the analytic continuation of the hyperbolic Schwarz map) to the adjacent sector $\Sigma_{2}$. In this study, a Stokes multiplier of $(E)$ comes in. In $\S 4.1$ we write down the general formula of the location of such limit points in terms of Stokes multipliers. In $\S 4.2$ and $\S 4.3$ we discuss some special cases in which the Stokes multipliers are known. 


\subsection{General case}

As a preparation, we consider the image of a ray in $\Sigma_{p}$ under $\mathscr{S}$ with an arbitrary basis $\left(v_{0}, v_{1}\right)$. Such a basis $\left(v_{0}, v_{1}\right)$ can be expressed as a linear combination of $\left(u_{+}^{(p)}, u_{-}^{(p)}\right)$ as

$$
\left(\begin{array}{l}
v_{0} \\
v_{1}
\end{array}\right)=\left(\begin{array}{ll}
a & b \\
c & d
\end{array}\right)\left(\begin{array}{l}
u_{+}^{(p)} \\
u_{-}^{(p)}
\end{array}\right) \quad(a d-b c \neq 0) .
$$

Let $\AA$ be a direction which is not a singular direction. We assume

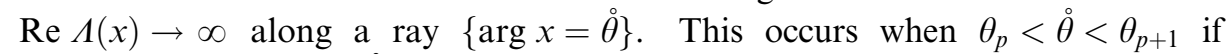
$p$ is even, and $\theta_{p-1}<\stackrel{\circ}{0}<\theta_{p}$ if $p$ is odd (cf. Remark 2.8). As we saw in the previous section (cf. Theorem 3.9 (I)), the image of the ray under $\mathscr{S}$ with respect to $\left(u_{+}^{(p)}, u_{-}^{(p)}\right)$ tends to the north pole $(0,0,1)$. To determine where this point is mapped to under the transformation (4.1), we use

Lemma 4.1 ([SY, Lemma 2.2]). Let $\left\{v_{0}, v_{1}\right\}$ and $\left\{u_{0}, u_{1}\right\}$ be two sets of independent solutions of the equation $(E)$ that are related as $v_{0}=c_{1} u_{0}+c_{2} u_{1}$ and $v_{1}=c_{3} u_{0}+c_{4} u_{1}$. Let $\left(y_{1}, y_{2}, y_{3}\right)$ (resp. $\left.\left(Y_{1}, Y_{2}, Y_{3}\right)\right)$ denote the coordinates of the image of the point $x$ under the hyperbolic Schwarz map defined by use of $\left\{v_{0}, v_{1}\right\}$ (resp. $\left\{u_{0}, u_{1}\right\}$ ). Then both coordinates are related as follows.

$$
\begin{aligned}
& y_{1}=\frac{A^{0}\left(1+|Y|^{2}\right)+2 A^{1} Y_{1}+2 A^{2} Y_{2}+2 A^{3} Y_{3}}{2 c\left(1-|Y|^{2}\right)+D^{0}\left(1+|Y|^{2}\right)+2 D^{1} Y_{1}+2 D^{2} Y_{2}+2 D^{3} Y_{3}}, \\
& y_{2}=\frac{B^{0}\left(1+|Y|^{2}\right)+2 B^{1} Y_{1}+2 B^{2} Y_{2}+2 B^{3} Y_{3}}{2 c\left(1-|Y|^{2}\right)+D^{0}\left(1+|Y|^{2}\right)+2 D^{1} Y_{1}+2 D^{2} Y_{2}+2 D^{3} Y_{3}}, \\
& y_{3}=\frac{C^{0}\left(1+|Y|^{2}\right)+2 C^{1} Y_{1}+2 C^{2} Y_{2}+2 C^{3} Y_{3}}{2 c\left(1-|Y|^{2}\right)+D^{0}\left(1+|Y|^{2}\right)+2 D^{1} Y_{1}+2 D^{2} Y_{2}+2 D^{3} Y_{3}},
\end{aligned}
$$

where $c=\left|c_{1} c_{4}-c_{2} c_{3}\right|,|Y|^{2}=Y_{1}^{2}+Y_{2}^{2}+Y_{3}^{2}$ and

$$
\begin{array}{ll}
A^{0}=c_{1} \overline{c_{3}}+\overline{c_{1}} c_{3}+c_{2} \overline{c_{4}}+\overline{c_{2}} c_{4}, & A^{1}=c_{1} \overline{c_{4}}+\overline{c_{1}} c_{4}+c_{2} \overline{c_{3}}+\overline{c_{2}} c_{3}, \\
A^{2}=i\left(-c_{1} \overline{c_{4}}+\overline{c_{1}} c_{4}+c_{2} \overline{c_{3}}-\overline{c_{2}} c_{3}\right), & A^{3}=c_{1} \overline{c_{3}}+\overline{c_{1}} c_{3}-c_{2} \overline{c_{4}}-\overline{c_{2}} c_{4}, \\
B^{0}=i\left(c_{1} \overline{c_{3}}-\overline{c_{1}} c_{3}+c_{2} \overline{c_{4}}-\overline{c_{2}} c_{4}\right), & B^{1}=i\left(c_{1} \overline{c_{4}}-\overline{c_{1}} c_{4}+c_{2} \overline{c_{3}}-\overline{c_{2}} c_{3}\right), \\
B^{2}=c_{1} \overline{c_{4}}+\overline{c_{1}} c_{4}-c_{2} \overline{c_{3}}-\overline{c_{2}} c_{3}, & B^{3}=i\left(c_{1} \overline{c_{3}}-\overline{c_{1}} c_{3}-c_{2} \overline{c_{4}}+\overline{c_{2}} c_{4}\right), \\
C^{0}=c_{1} \overline{c_{1}}+\overline{c_{2}} c_{2}-c_{3} \overline{c_{3}}-\overline{c_{4}} c_{4}, & C^{1}=c_{1} \overline{c_{2}}+\overline{c_{1}} c_{2}-c_{3} \overline{c_{4}}-\overline{c_{3}} c_{4}, \\
C^{2}=i\left(-c_{1} \overline{c_{2}}+\overline{c_{1}} c_{2}+c_{3} \overline{c_{4}}-\overline{c_{3}} c_{4}\right), & C^{3}=c_{1} \overline{c_{1}}-\overline{c_{2}} c_{2}-c_{3} \overline{c_{3}}+\overline{c_{4}} c_{4},
\end{array}
$$




$$
\begin{array}{ll}
D^{0}=c_{1} \overline{c_{1}}+\overline{c_{2}} c_{2}+c_{3} \overline{c_{3}}+\overline{c_{4}} c_{4}, & D^{1}=c_{1} \overline{c_{2}}+\overline{c_{1}} c_{2}+c_{3} \overline{c_{4}}+\overline{c_{3}} c_{4}, \\
D^{2}=i\left(-c_{1} \overline{c_{2}}+\overline{c_{1}} c_{2}-c_{3} \overline{c_{4}}+\overline{c_{3}} c_{4}\right), & D^{3}=c_{1} \overline{c_{1}}-\overline{c_{2}} c_{2}+c_{3} \overline{c_{3}}-\overline{c_{4}} c_{4} .
\end{array}
$$

If we put

$$
Y_{1}=Y_{2}=0, \quad Y_{3}=1, \quad c_{1}=a, \quad c_{2}=b, \quad c_{3}=c, \quad c_{4}=d,
$$

we obtain

$$
\begin{aligned}
A^{0}+A^{3}=4 \operatorname{Re}(\bar{a} c), & B^{0}+B^{3}=4 \operatorname{Im}(\bar{a} c), \\
C^{0}+C^{3}=2\left(|a|^{2}-|c|^{2}\right), & D^{0}+D^{3}=2\left(|a|^{2}+|c|^{2}\right) .
\end{aligned}
$$

Therefore the north pole $(0,0,1)$ is mapped to $\llbracket a, c \rrbracket$. Here we introduce the symbol

$$
\llbracket \alpha, \beta \rrbracket:=\left(\frac{2 \operatorname{Re}(\bar{\alpha} \beta)}{|\alpha|^{2}+|\beta|^{2}}, \frac{2 \operatorname{Im}(\bar{\alpha} \beta)}{|\alpha|^{2}+|\beta|^{2}}, \frac{|\alpha|^{2}-|\beta|^{2}}{|\alpha|^{2}+|\beta|^{2}}\right)
$$

to locate a point on the boundary sphere of $\mathbf{B}^{3}$. The other case, that is, the case when $\operatorname{Re} \Lambda(x) \rightarrow-\infty$ can be treated in the same way. Thus we obtain

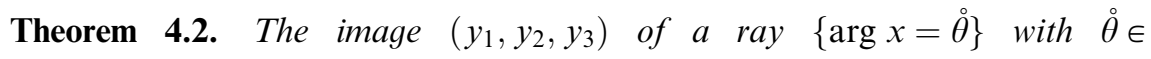
$\left(\theta_{p-1}, \theta_{p}\right) \cup\left(\theta_{p}, \theta_{p+1}\right)$ under the hyperbolic Schwarz map $\mathscr{S}$ with a basis $\left(v_{0}, v_{1}\right)$ defined in (4.1) behaves as follows:

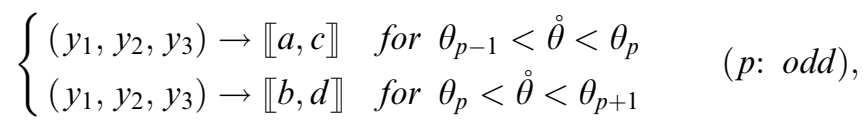

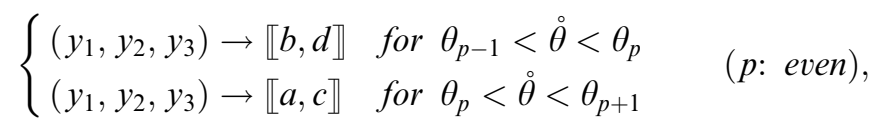

as $x$ tends to infinity (see Figure 8).

Now we find the behavior of the image of a ray under $\mathscr{S}$ in the adjacent sector $\Sigma_{p+1}$. Let us consider the case when $p$ is an odd integer. From Theorem 4.2 we already know that the image of a ray $\{\arg x=\ddot{\theta}\}$ with $\theta_{p}<\stackrel{\circ}{\theta}<\theta_{p+1}$ tends to $\llbracket b, d \rrbracket$ as $x$ tends to infinity along that ray. To consider the case $\theta_{p+1}<\stackrel{\circ}{\theta}<\theta_{p+2}$, we recall the basis $\left(u_{+}^{(p)}, u_{-}^{(p)}\right)$ and $\left(u_{+}^{(p+1)}, u_{-}^{(p+1)}\right)$ are related by

$$
\left(\begin{array}{l}
u_{+}^{(p)} \\
u_{-}^{(p)}
\end{array}\right)=\left(\begin{array}{cc}
1 & 0 \\
T_{p+1} & 1
\end{array}\right)\left(\begin{array}{l}
u_{+}^{(p+1)} \\
u_{-}^{(p+1)}
\end{array}\right)
$$



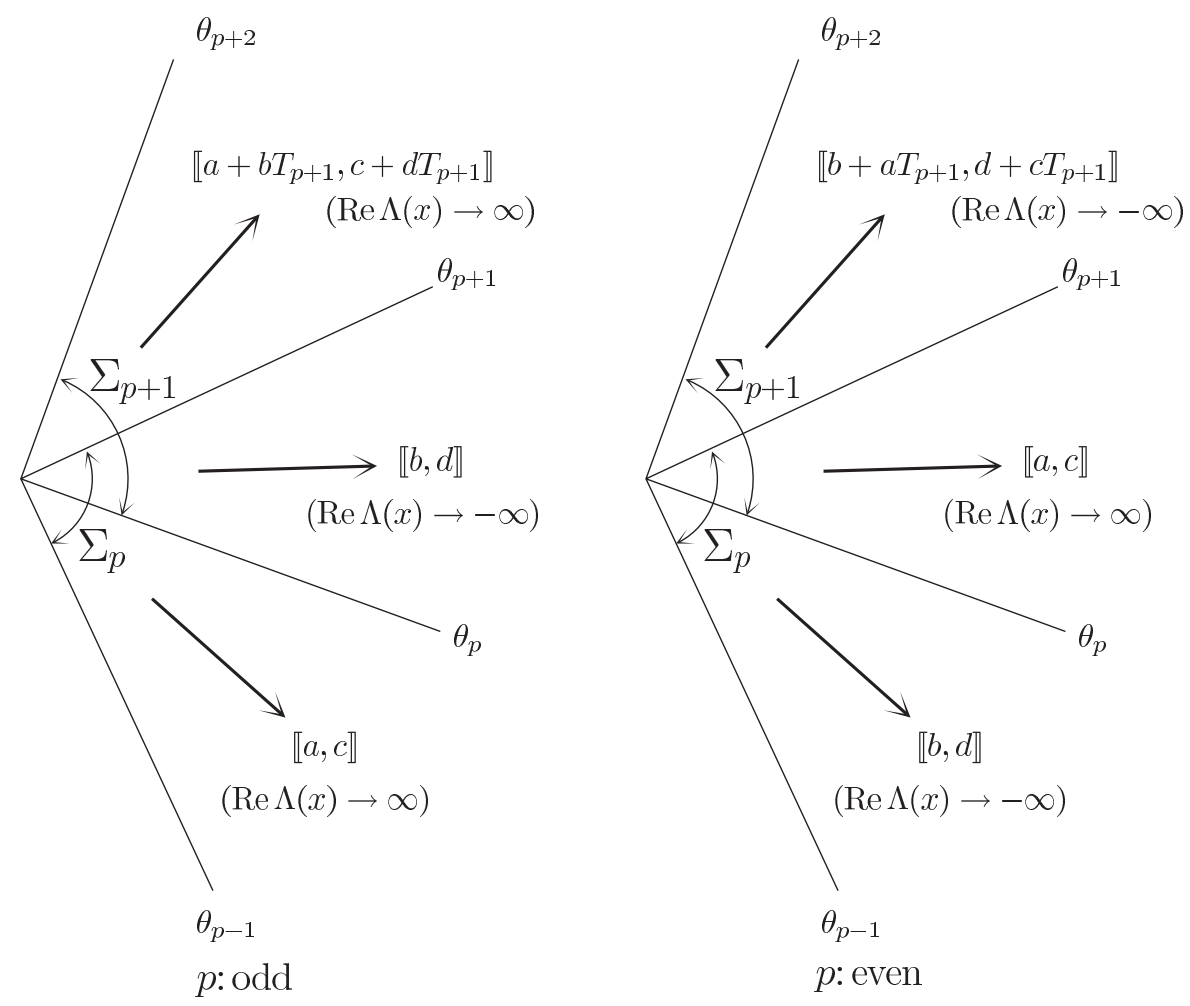

Fig. 8. The image of the ray under the hyperbolic Schwarz map with the basis $\left(v_{0}, v_{1}\right)$ defined in (4.1). (Left: $p$ is even, Right: $p$ is odd)

with a Stokes multiplier $T_{p+1}$ (cf. Theorem 2.9). Hence we obtain

$$
\left(\begin{array}{l}
v_{0} \\
v_{1}
\end{array}\right)=\left(\begin{array}{ll}
a & b \\
c & d
\end{array}\right)\left(\begin{array}{cc}
1 & 0 \\
T_{p+1} & 1
\end{array}\right)\left(\begin{array}{l}
u_{+}^{(p+1)} \\
u_{-}^{(p+1)}
\end{array}\right)=\left(\begin{array}{ll}
a+b T_{p+1} & b \\
c+d T_{p+1} & d
\end{array}\right)\left(\begin{array}{l}
u_{+}^{(p+1)} \\
u_{-}^{(p+1)}
\end{array}\right) .
$$

Therefore it follows from Theorem 4.2 again that the image of a ray

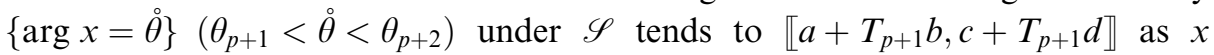
tends to infinity along the ray. The case where $p$ is even can be treated in a similar way. Thus we arrive at

Theorem 4.3. If $x$ tends to infinity along a ray $\left\{\arg x=\stackrel{\theta}{\}}\right.$ with $\theta_{p+1}<$ $\stackrel{\ominus}{\theta}<\theta_{p+2}$, then its image under the hyperbolic Schwarz map $\mathscr{S}$ tends to $\llbracket a+T_{p+1} b, c+T_{p+1} d \rrbracket$ if $p$ is odd and tends to $\llbracket b+T_{p+1} a, d+T_{p+1} c \rrbracket$ if $p$ is even. (See Figure 8).

As a corollary of Theorem 4.2 and Theorem 4.3, we obtain

Corollary 4.4. Let $\llbracket \alpha_{p-1}, \beta_{p-1} \rrbracket\left(\right.$ resp., $\left.\llbracket \alpha_{p}, \beta_{p} \rrbracket\right)$ be the limit point of the image of a ray in $\Sigma_{p-1} \cap \Sigma_{p}$ (resp., in $\Sigma_{p} \cap \Sigma_{p+1}$ ) under the hyperbolic Schwarz 
map $\mathscr{S}$ with some fixed basis of solutions of $(E)$. Then the limit point of the image of a ray in $\Sigma_{p+1} \cap \Sigma_{p+2}$ is given by $\llbracket \alpha_{p+1}, \beta_{p+1} \rrbracket$, where

$$
\left(\begin{array}{c}
\alpha_{p+1} \\
\beta_{p+1}
\end{array}\right)=T_{p+1}\left(\begin{array}{c}
\alpha_{p} \\
\beta_{p}
\end{array}\right)+\left(\begin{array}{c}
\alpha_{p-1} \\
\beta_{p-1}
\end{array}\right) .
$$

By repeated use of Corollary 4.4 we can locate the limit point of the image of a ray in each sectors. For example if we choose

$$
\left(\begin{array}{l}
v_{0} \\
v_{1}
\end{array}\right)=\left(\begin{array}{ll}
a & b \\
c & d
\end{array}\right)\left(\begin{array}{c}
u_{+}^{(1)} \\
u_{-}^{(1)}
\end{array}\right) \quad(a d-b c \neq 0)
$$

as a basis of solutions of $(E)$, we can determine the limit point of the image of a ray by solving $(4.3)$ with $\left(\alpha_{0}, \beta_{0}\right)=(a, c)$ and $\left(\alpha_{1}, \beta_{1}\right)=(b, d)$.

It is, however, difficult to determine Stokes multipliers $\left\{T_{p}\right\}$ for a general coefficient $q(x)$. In the following subsections, we consider the special case in which Stokes multipliers are known.

\subsection{Example: the Airy equation}

We consider the case $q(x)=x$, i.e., the Airy equation. In this case the Stokes multiplier $T_{p}$ is equal to $i$ for every integer $p$ (cf. Proposition A.2). Let us compute the limit point of the image of a ray under the hyperbolic Schwarz map. As a basis of solutions of $(E)$ we choose (4.4). Then it follows from Corollary 4.4 that the limit point of a ray in

$$
\mathcal{S}_{p}=\Sigma_{p} \cap \Sigma_{p+1}=\left\{x ; \theta_{p}<\arg x<\theta_{p+1}\right\}
$$

is given as follows:

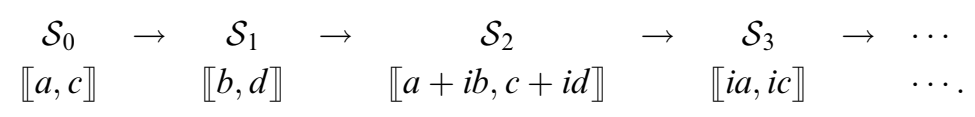

Since $\llbracket i a, i c \rrbracket=\llbracket a, c \rrbracket$ holds, the limit point of the image of a ray in $\mathcal{S}_{3}$ coincides with that in $\mathcal{S}_{0}$. This is what we expect since every solution of the Airy equation is entire. Let us compare our result with that obtained in $[\mathrm{SY}]$. In $[\mathrm{SY}]$, the location of a limit point of the image of a ray is determined by using the Airy functions $\operatorname{Ai}(x)$ and $\operatorname{Bi}(x)$. So we express the Airy functions as a linear combination of our solution $u_{ \pm}^{(1)}$.

Lemma 4.5. We obtain

$$
\left(\begin{array}{l}
\operatorname{Ai}(x) \\
\operatorname{Bi}(x)
\end{array}\right)=\frac{1}{2 \sqrt{\pi}}\left(\begin{array}{ll}
0 & 1 \\
2 & i
\end{array}\right)\left(\begin{array}{l}
u_{+}^{(1)}(x) \\
u_{-}^{(1)}(x)
\end{array}\right) .
$$


Proof. Since $\operatorname{Ai}(x)$ and $\operatorname{Bi}(x)$ admit the asymptotic expansions:

$$
\begin{aligned}
& \operatorname{Ai}(x)=\frac{1}{2 \sqrt{\pi}} e^{-2 x^{3 / 2} / 3}\left(1+O\left(|x|^{-1 / 2}\right)\right) \\
& \operatorname{Bi}(x)=\frac{1}{\sqrt{\pi}} e^{2 x^{3 / 2} / 3}\left(1+O\left(|x|^{-1 / 2}\right)\right)
\end{aligned}
$$

as $x \rightarrow \infty(-\pi / 3<\arg x<\pi / 3)$, we find that $\operatorname{Ai}(x) \ll \operatorname{Bi}(x)$ in $\mathcal{S}_{0}$. Hence $\operatorname{Ai}(x)$, which is the exponentially small solution, should coincide with $a u_{-}^{(1)}(x)$ for some $a \in C$. Comparing the asymptotic behaviors of $\mathrm{Ai}(x)$ and $u_{-}^{(1)}(x)$ as $x \rightarrow+\infty$, we have $a=1 / 2 \sqrt{\pi}$. By a similar argument, we also obtain

$$
\operatorname{Bi}(x)=\frac{1}{\sqrt{\pi}}\left(u_{+}^{(1)}(x)+c u_{-}^{(1)}(x)\right)
$$

with some constant $c$. To determine this constant $c$, we next consider the asymptotic behavior for $x=-r, r \rightarrow \infty$. The Airy functions behave as

$$
\begin{aligned}
\operatorname{Ai}(-r) & =\frac{1}{\sqrt{\pi}} r^{-1 / 4} \cos \left(\frac{2}{3} r^{3 / 2}-\frac{\pi}{4}\right)\left(1+O\left(r^{-1 / 2}\right)\right) \\
& =\frac{e^{-i \pi / 4}}{2 \sqrt{\pi}} r^{-1 / 4}\left[e^{2 i r^{3 / 2} / 3}+i e^{-2 i r^{3 / 2} / 3}\right]\left(1+O\left(r^{-1 / 2}\right)\right) \\
\operatorname{Bi}(-r) & =-\frac{1}{\sqrt{\pi}} r^{-1 / 4} \sin \left(\frac{2}{3} r^{3 / 2}-\frac{\pi}{4}\right)\left(1+O\left(r^{-1 / 2}\right)\right) \\
& =-\frac{e^{-i \pi / 4}}{2 i \sqrt{\pi}} r^{-1 / 4}\left[e^{2 i r^{3 / 2} / 3}-i e^{-2 i r^{3 / 2} / 3}\right]\left(1+O\left(r^{-1 / 2}\right)\right)
\end{aligned}
$$

in this limit. On the other hand, by using $u_{+}^{(1)}(x)=u_{+}^{(2)}(x)$ and $u_{-}^{(1)}(x)=$ $u_{-}^{(2)}(x)+i u_{+}^{(2)}(x)$, we obtain

$$
\begin{aligned}
\operatorname{Ai}(-r) & =\frac{1}{2 \sqrt{\pi}}\left(u_{-}^{(2)}(-r)+i u_{+}^{(2)}(-r)\right) \\
& =\frac{e^{-i \pi / 4}}{2 \sqrt{\pi}} r^{-1 / 4}\left[e^{2 i r^{3 / 2} / 3}+i e^{-2 i r^{3 / 2} / 3}\right]\left(1+O\left(r^{-1 / 2}\right)\right), \\
\operatorname{Bi}(-r) & =\frac{1}{\sqrt{\pi}}\left((1+i c) u_{+}^{(2)}(-r)+c u_{-}^{(2)}(-r)\right) \\
& =\frac{e^{-i \pi / 4}}{\sqrt{\pi}}\left[(1+i c) e^{-2 i r^{3 / 2} / 3}+c e^{2 i r^{3 / 2} / 3}\right]\left(1+O\left(r^{-1 / 2}\right)\right) .
\end{aligned}
$$

Therefore $c=i / 2$. 
If we use $(\operatorname{Ai}(x), \operatorname{Bi}(x))$ as a basis, (4.5) becomes

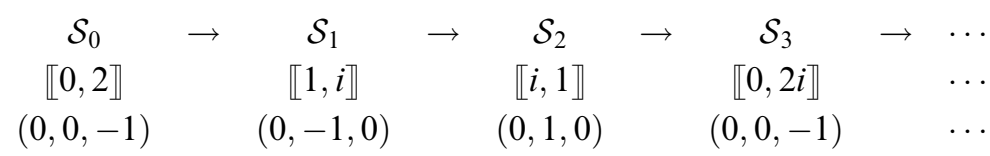

This agrees with the result in $[\mathrm{SY}]$.

\subsection{Example: the equation with $q(x)=x^{n}$}

As the last example we consider the case $q(x)=x^{n}$. In this case

$$
\hat{u}_{ \pm}=\exp \left[ \pm \frac{1}{n / 2+1} x^{n / 2+1}\right] x^{-n / 4}\left(1+O\left(|x|^{-1}\right)\right),
$$

and Stokes multipliers are given by

$$
T_{p}=T=2 i \sin \left(\frac{n \pi}{2 n+4}\right) \quad(p \in \boldsymbol{Z}) .
$$

(Cf. Proposition A.4. Note that all Stokes multipliers are the same.) Let us chose a basis $\left(v_{0}, v_{1}\right)$ as in (4.4). Then the limit point of the image of a ray moves as follows:

$$
\begin{aligned}
& \mathcal{S}_{0} \quad \rightarrow \quad \mathcal{S}_{1} \quad \rightarrow \quad \mathcal{S}_{2} \\
& \llbracket a, c \rrbracket \quad \llbracket b, d \rrbracket \quad \llbracket a+T b, c+T d \rrbracket
\end{aligned}
$$

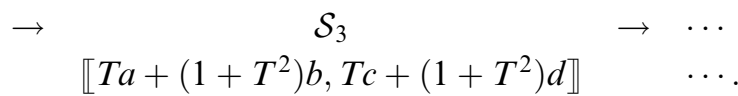

Let us see some special cases.

- $q(x)=x^{2}:$ In this case $T=i \sqrt{2}$, and a limit point of the image of a ray moves as follows:

$$
\begin{aligned}
& \begin{array}{llllll}
\mathcal{S}_{0} & \rightarrow & \mathcal{S}_{1} & \rightarrow & \mathcal{S}_{2}
\end{array} \\
& \llbracket 1,0 \rrbracket \quad \llbracket 0,1 \rrbracket \quad \llbracket 1, i \sqrt{2} \rrbracket \\
& (0,0,1) \quad(0,0,-1) \quad\left(0, \frac{2 \sqrt{2}}{3},-\frac{1}{3}\right) \\
& \rightarrow \quad \begin{array}{c}
\mathcal{S}_{3} \\
\llbracket i \sqrt{2},-1 \rrbracket
\end{array} \quad \rightarrow \quad \begin{array}{c}
\mathcal{S}_{4} \\
\llbracket-1,0 \rrbracket
\end{array} \quad \rightarrow \quad \cdots \\
& \left(0, \frac{2 \sqrt{2}}{3}, \frac{1}{3}\right) \quad(0,0,1) \quad \cdots \text {. }
\end{aligned}
$$


- $q(x)=x^{4}:$ In this case $T=i \sqrt{3}$, and a limit point of the image of a ray moves as follows:



$$
\begin{aligned}
& (0,0,1) \quad(0,0,-1) \quad\left(0, \frac{\sqrt{3}}{2},-\frac{1}{2}\right) \quad\left(0, \frac{4 \sqrt{3}}{7},-\frac{1}{7}\right)
\end{aligned}
$$

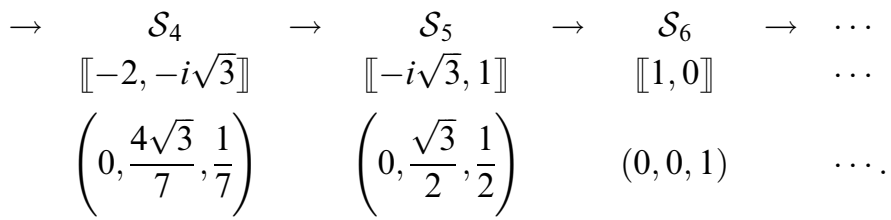

To describe the location of the limit point for general $n$ we introduce the following matrices:

$$
J_{n}=\left\{\left(\begin{array}{ll}
1 & 0 \\
T & 1
\end{array}\right)\left(\begin{array}{ll}
1 & T \\
0 & 1
\end{array}\right)\right\}^{n}=\left(\begin{array}{cc}
1 & T \\
T & 1+T^{2}
\end{array}\right)^{n} .
$$

Theorem 4.6. The limit point of the image of a ray $\{\arg x=\grave{\theta}\}$ with $\theta_{p}<\stackrel{\circ}{0} \theta_{p+1}$ (i.e., $x \in \mathcal{S}_{p}$ ) under the hyperbolic Schwarz map is given by $\llbracket \alpha_{p}, \beta_{p} \rrbracket$, where

$$
\left(\begin{array}{l}
\alpha_{2 l-1} \\
\beta_{2 l-1}
\end{array}\right)=\left(\begin{array}{ll}
a & b \\
c & d
\end{array}\right) J_{l-1}\left(\begin{array}{l}
0 \\
1
\end{array}\right), \quad\left(\begin{array}{l}
\alpha_{2 l} \\
\beta_{2 l}
\end{array}\right)=\left(\begin{array}{ll}
a & b \\
c & d
\end{array}\right) J_{l-1}\left(\begin{array}{ll}
1 & 0 \\
T & 1
\end{array}\right)\left(\begin{array}{l}
1 \\
0
\end{array}\right)
$$

for $l=1,2, \ldots$.

Proof. We prove our assertion by induction on $l$. The case $l=1$ is already shown. We now prove our assertion for $l \geq 2$. It follows from Corollary 4.4 and the hypothesis of the induction that

$$
\begin{aligned}
\left(\begin{array}{l}
\alpha_{2 l+1} \\
\beta_{2 l+1}
\end{array}\right) & =\left(\begin{array}{l}
\alpha_{2 l-1} \\
\beta_{2 l-1}
\end{array}\right)+T\left(\begin{array}{l}
\alpha_{2 l} \\
\beta_{2 l}
\end{array}\right)=\left(\begin{array}{ll}
a & b \\
c & d
\end{array}\right) J_{l-1}\left\{\left(\begin{array}{l}
0 \\
1
\end{array}\right)+T\left(\begin{array}{l}
1 \\
T
\end{array}\right)\right\} \\
& =\left(\begin{array}{ll}
a & b \\
c & d
\end{array}\right) J_{l-1}\left(\begin{array}{c}
T \\
1+T^{2}
\end{array}\right)=\left(\begin{array}{ll}
a & b \\
c & d
\end{array}\right) J_{l-1}\left(\begin{array}{cc}
1 & T \\
T & 1+T^{2}
\end{array}\right)\left(\begin{array}{l}
0 \\
1
\end{array}\right) \\
& =\left(\begin{array}{ll}
a & b \\
c & d
\end{array}\right) J_{l}\left(\begin{array}{l}
0 \\
1
\end{array}\right) .
\end{aligned}
$$

In a similar way we find

$$
\begin{aligned}
\left(\begin{array}{c}
\alpha_{2 l+2} \\
\beta_{2 l+2}
\end{array}\right) & =\left(\begin{array}{l}
\alpha_{2 l} \\
\beta_{2 l}
\end{array}\right)+T\left(\begin{array}{l}
\alpha_{2 l+1} \\
\beta_{2 l+1}
\end{array}\right) \\
& =\left(\begin{array}{ll}
a & b \\
c & d
\end{array}\right) J_{l-1}\left\{\left(\begin{array}{ll}
1 & 0 \\
T & 1
\end{array}\right)\left(\begin{array}{l}
1 \\
0
\end{array}\right)+T\left(\begin{array}{cc}
1 & T \\
T & 1+T^{2}
\end{array}\right)\left(\begin{array}{l}
0 \\
1
\end{array}\right)\right\}
\end{aligned}
$$




$$
\begin{aligned}
& =\left(\begin{array}{ll}
a & b \\
c & d
\end{array}\right) J_{l-1}\left(\begin{array}{c}
1+T^{2} \\
2 T+T^{3}
\end{array}\right) \\
& =\left(\begin{array}{ll}
a & b \\
c & d
\end{array}\right) J_{l-1}\left(\begin{array}{cc}
1+T^{2} & T \\
2 T+T^{3} & 1+T^{2}
\end{array}\right)\left(\begin{array}{l}
1 \\
0
\end{array}\right) \\
& =\left(\begin{array}{ll}
a & b \\
c & d
\end{array}\right) J_{l-1}\left(\begin{array}{cc}
1 & T \\
T & 1+T^{2}
\end{array}\right)\left(\begin{array}{ll}
1 & 0 \\
T & 1
\end{array}\right)\left(\begin{array}{l}
1 \\
0
\end{array}\right) .
\end{aligned}
$$

This completes the proof.

As an application of this theorem we confirm the following:

Proposition 4.7. For every $n \geq 1$, we have $\llbracket \alpha_{n+2}, \beta_{n+2} \rrbracket=\llbracket \alpha_{0}, \beta_{0} \rrbracket$.

This relation should be, of course, satisfied, for the following reasons: $\theta_{0} \equiv \theta_{n+2} \bmod 2 \pi$, and the projections of sectors $\Sigma_{0}$ and $\Sigma_{n+2}$ to the complex plane are the same, and every solution of $(E)$ with $q(x)=x^{n}$ is entire. We confirm this relation by using Theorem 4.6. We divide the proof into two cases.

The case $n=2 m-1$ : Since the Stokes multiplier $T$ satisfies (A.3), we obtain

$$
\left(\begin{array}{ll}
1 & T \\
0 & 1
\end{array}\right) J_{m}=(-1)^{m+1}\left(\begin{array}{ll}
0 & i \\
i & 0
\end{array}\right)
$$

or,

$$
J_{m}=(-1)^{m+1}\left(\begin{array}{cc}
1 & -T \\
0 & 1
\end{array}\right)\left(\begin{array}{ll}
0 & i \\
i & 0
\end{array}\right)=(-1)^{m+1}\left(\begin{array}{cc}
-i T & i \\
i & 0
\end{array}\right)
$$

Hence

$$
\left(\begin{array}{c}
\alpha_{2 m+1} \\
\beta_{2 m+1}
\end{array}\right)=\left(\begin{array}{ll}
a & b \\
c & d
\end{array}\right) J_{m}\left(\begin{array}{l}
0 \\
1
\end{array}\right)=(-1)^{m+1}\left(\begin{array}{c}
i a \\
i c
\end{array}\right) .
$$

Therefore we obtain

$$
\llbracket \alpha_{2 m+1}, \beta_{2 m+1} \rrbracket=\llbracket(-1)^{m+1} i a,(-1)^{m+1} i c \rrbracket=\llbracket a, c \rrbracket=\llbracket \alpha_{0}, \beta_{0} \rrbracket .
$$

The case $n=2 m$ : Since the Stokes multiplier $T$ in this case satisfies (A.4), we obtain

$$
\left(\begin{array}{ll}
1 & T \\
0 & 1
\end{array}\right) J_{m}\left(\begin{array}{ll}
1 & 0 \\
T & 1
\end{array}\right)=(-1)^{m}\left(\begin{array}{ll}
1 & 0 \\
0 & 1
\end{array}\right)
$$

therefore

$$
J_{m}\left(\begin{array}{ll}
1 & 0 \\
T & 1
\end{array}\right)=(-1)^{m}\left(\begin{array}{cc}
1 & -T \\
0 & 1
\end{array}\right)
$$


Hence we obtain

$$
\left(\begin{array}{l}
\alpha_{2 m+2} \\
\beta_{2 m+2}
\end{array}\right)=\left(\begin{array}{c}
(-1)^{m} a \\
(-1)^{m} c
\end{array}\right)
$$

and

$$
\llbracket \alpha_{2 m+2}, \beta_{2 m+2} \rrbracket=\llbracket(-1)^{m} a,(-1)^{m} c \rrbracket=\llbracket a, c \rrbracket=\llbracket \alpha_{0}, \beta_{0} \rrbracket .
$$

Thus the proof of Proposition 4.7 is completed.

\section{A. Determination of Stokes multipliers}

In this appendix, to make our paper self-contained, we summarize several properties of Stokes multipliers for $q(x)=x^{n}$ with proofs.

\section{A.1. Some relations of Stokes multipliers for $q(x)=x^{n}$}

If the coefficient $q(x)$ is just a monomial $x^{n},(E)$ does not change under the rotation $x \mapsto e^{-2 i \pi /(n+2)} x$ of the independent variable. In this case all the Stokes multipliers $T_{p}(p \in \boldsymbol{Z})$ coincides to each other; this is what we want to show in this subsection.

As an illustration we consider the case $q(x)=x$, i.e., the Airy equation. Let $\left\{u_{ \pm}^{(p)}\right\}$ be solutions specified in Theorem 2.4.

Proposition A.1. The solutions $\left\{u_{ \pm}^{(p)}\right\}$ of the Airy equation are related as

$$
u_{ \pm}^{(p)}(x)=e^{-i \pi / 6} u_{\mp}^{(p-1)}\left(e^{-2 i \pi / 3} x\right) \quad \text { in } \Sigma_{p} .
$$

Proof. We give a proof of our claim for $p=1$ (The other cases are similarly done). Let $v_{ \pm}(x)=u_{ \pm}^{(0)}\left(e^{-2 i \pi / 3} x\right)$. This $v_{ \pm}(x)$ is also a solution of the Airy equation, since the Airy equation does not change under the rotation $x \mapsto e^{-2 i \pi / 3} x$. Furthermore it follows from this relation that the asymptotic behavior of $v_{ \pm}(x)$ as $x$ tends to infinity with $x \in \Sigma_{1}$ is given by

$$
v_{ \pm}(x) \sim \hat{u}_{ \pm}\left(e^{-2 i \pi / 3} x\right)=e^{i \pi / 6} \exp \left[\mp \frac{2}{3} x^{3 / 2}\right] x^{-1 / 4}\left(1+O\left(|x|^{-1 / 2}\right)\right),
$$

since $e^{-2 i \pi / 3} x$ is in $\Sigma_{0}$. Then the uniqueness of the asymptotic expansion in $\Sigma_{1}$ guarantees that

$$
v_{ \pm}(x)=e^{i \pi / 6} u_{\mp}^{(1)}(x) .
$$

This completes the proof.

Armed with this proposition we now show that all the Stokes multipliers are the same. By (2.16),

$$
u_{+}^{(0)}(x)=u_{+}^{(1)}(x)+T_{1} u_{-}^{(1)}(x), \quad u_{-}^{(0)}(x)=u_{-}^{(1)}(x)
$$


in $\Sigma_{0} \cap \Sigma_{1}$. We then substitute $e^{-2 i \pi / 3} x$ into the above relations to obtain

$$
\begin{aligned}
& u_{+}^{(0)}\left(e^{-2 i \pi / 3} x\right)=u_{+}^{(1)}\left(e^{-2 i \pi / 3} x\right)+T_{1} u_{-}^{(1)}\left(e^{-2 i \pi / 3} x\right), \\
& u_{-}^{(0)}\left(e^{-2 i \pi / 3} x\right)=u_{-}^{(1)}\left(e^{-2 i \pi / 3} x\right) .
\end{aligned}
$$

It follows from these relations and Proposition A.1 that

$$
u_{-}^{(1)}(x)=u_{-}^{(2)}(x)+T_{1} u_{+}^{(2)}(x), \quad u_{+}^{(1)}(x)=u_{+}^{(2)}(x) .
$$

This means that $T_{2}=T_{1}(=: T)$. In the same way we obtain $T_{p}=T$ for every integer $p$.

Furthermore we can determine $T$ as follows: since all of the Stokes multiplies are equal to $T$, we obtain

$$
\begin{aligned}
\left(\begin{array}{l}
u_{+}^{(0)}(x) \\
u_{-}^{(0)}(x)
\end{array}\right) & =\left(\begin{array}{ll}
1 & T \\
0 & 1
\end{array}\right)\left(\begin{array}{ll}
1 & 0 \\
T & 1
\end{array}\right)\left(\begin{array}{ll}
1 & T \\
0 & 1
\end{array}\right)\left(\begin{array}{c}
u_{+}^{(3)}(x) \\
u_{-}^{(3)}(x)
\end{array}\right) \\
& =\left(\begin{array}{ll}
1 & T \\
0 & 1
\end{array}\right)\left(\begin{array}{ll}
1 & 0 \\
T & 1
\end{array}\right)\left(\begin{array}{ll}
1 & T \\
0 & 1
\end{array}\right)\left(\begin{array}{cc}
0 & -i \\
-i & 0
\end{array}\right)\left(\begin{array}{l}
u_{+}^{(0)}\left(x e^{-2 i \pi}\right) \\
u_{-}^{(0)}\left(x e^{-2 i \pi}\right)
\end{array}\right) \quad\left(x \in \Sigma_{3}\right) .
\end{aligned}
$$

Here we have used the relation between $\left\{u_{ \pm}^{(0)}\right\}$ and $\left\{u_{ \pm}^{(3)}\right\}$ (cf. Remark 2.6). On the other hand, since solutions of $(E)$ with $q(x)=x$ are single-valued near the infinity, we obtain $u_{ \pm}^{(0)}\left(x e^{-2 i \pi}\right)=u_{ \pm}^{(0)}(x)$. Hence we obtain the following relation for $T$ :

$$
\left(\begin{array}{ll}
1 & T \\
0 & 1
\end{array}\right)\left(\begin{array}{ll}
1 & 0 \\
T & 1
\end{array}\right)\left(\begin{array}{ll}
1 & T \\
0 & 1
\end{array}\right)=\left(\begin{array}{ll}
0 & i \\
i & 0
\end{array}\right)
$$

Eq. (A.1) has a unique solution: $T=i$. Therefore we obtain

Proposition A.2. If $q(x)=x$, then $T_{p}=i$ for every $p \in \boldsymbol{Z}$.

We now consider the case $q(x)=x^{n}$. The following proposition can be shown by an argument similar to that for the Airy equation (see (4.6) for the formal solutions in this case):

Proposition A.3. We have the following relations among the solutions $\left\{u_{ \pm}^{(p)}\right\}$ of $(E)$ with $q(x)=x^{n}(n \geq-1)$ :

$$
u_{ \pm}^{(p)}(x)=e^{-i n \pi /(2 n+4)} u_{\overline{+}}^{(p-1)}\left(e^{-2 i \pi /(n+2)} x\right) \quad \text { in } \Sigma_{p} .
$$

By using this proposition, we can show that $T_{p}=T_{p^{\prime}}(=: T)$ for any integers $p$ and $p^{\prime}$. We can also derive equations which the Stokes multipliers $T$ satisfy (cf. (A.1)). The results are the following: if $n=2 m-1$ with $m \geq 1$, then

$$
\left(\begin{array}{ll}
1 & T \\
0 & 1
\end{array}\right) \overbrace{\left(\begin{array}{ll}
1 & 0 \\
T & 1
\end{array}\right)\left(\begin{array}{ll}
1 & T \\
0 & 1
\end{array}\right) \cdots\left(\begin{array}{ll}
1 & 0 \\
T & 1
\end{array}\right)\left(\begin{array}{ll}
1 & T \\
0 & 1
\end{array}\right)}^{2 m}=(-1)^{m+1}\left(\begin{array}{ll}
0 & i \\
i & 0
\end{array}\right),
$$


and if $n=2 m$ with $m \geq 0$, then

$$
\overbrace{\left(\begin{array}{ll}
1 & T \\
0 & 1
\end{array}\right)\left(\begin{array}{ll}
1 & 0 \\
T & 1
\end{array}\right) \cdots\left(\begin{array}{ll}
1 & 0 \\
T & 1
\end{array}\right)\left(\begin{array}{ll}
1 & T \\
0 & 1
\end{array}\right)}^{2 m+2}=(-1)^{m}\left(\begin{array}{ll}
1 & 0 \\
0 & 1
\end{array}\right) .
$$

For general $n$, however, these equations (A.3) and (A.4) do not determine $T$ uniquely. So we use another method to determine $T$ in the next subsection.

\section{A.2. Stokes multipliers for $q(x)=x^{n}$}

In this subsection we prove

Proposition A.4 ([Si, Theorem 23.1]). Let $n=-1,0,1, \ldots$ and $T_{p}$ the Stokes multipliers of $(E)$ with $q(x)=x^{n}$. Then

$$
T_{p}=2 i \sin \left(\frac{n \pi}{2 n+4}\right)=2 i \cos \left(\frac{\pi}{n+2}\right)
$$

holds for every integer $p$.

Remark A.5. The proof given here is essentially the same as in $[\mathrm{Si}]$, where the Stokes multiplier is determined by use of symmetry of the equation relying on a special choice of independent solutions of the equation as in [ $\mathrm{Si}$, Chapter 5, $\S 23]$. We use the solutions given in Section 2, for which Theorem 2.9 holds; this makes the proof a little simpler than that in $[\mathrm{Si}]$.

Proof. We prove this proposition for $p=1$ (the other cases are similarly proved). By substituting (A.2) with $p=1$ into the right-hand side of (2.16) with $p=0$, we have

$$
\begin{aligned}
& u_{+}(x)=\omega^{-n / 4}\left\{u_{-}\left(\omega^{-1} x\right)+T_{1} u_{+}\left(\omega^{-1} x\right)\right\}, \\
& u_{-}(x)=\omega^{-n / 4} u_{+}\left(\omega^{-1} x\right),
\end{aligned}
$$

where $\omega=\exp (2 i \pi /(n+2))$ and we omit the suffix $(0)$.

When $n \geq 0$, (A.5) and (A.6) give

$$
u_{+}(0)=\omega^{-n / 4}\left\{u_{-}(0)+T_{1} u_{+}(0)\right\}, \quad u_{-}(0)=\omega^{-n / 4} u_{+}(0) .
$$

Here we note that $u_{+}(0) \neq 0$; otherwise $u_{-}(0)=0$ follows from the second equation of (A.7), which contradicts the supposition that $\left(u_{+}, u_{-}\right)$is a basis of $(E)$. Hence from (A.7), we obtain

$$
1=\omega^{-n / 2}+T_{1} \omega^{-n / 4},
$$


i.e.,

$$
T_{1}=\omega^{n / 4}-\omega^{-n / 4}=2 i \sin \left(\frac{n \pi}{2(n+2)}\right) .
$$

When $n=-1$, the origin is a regular singular point. Even in this case, (A.7) makes sense if we regard $u_{+}(0)$ (resp., $\left.u_{-}(0)\right)$ as the limit value of $u_{+}(x)$ (resp., $\left.u_{-}(x)\right)$ when $x$ goes to zero with $|\arg x+\pi| \leq 2 \pi$, since the characteristic exponents at the origin are 0 and 1 . Then the same reasoning as before gives (A.8) with $n=-1$.

\section{References}

[SYY] Sasaki, T., Yamada, K. and Yoshida, M., Hyperbolic Schwarz map for the hypergeometric equation, Experiment. Math., 17 (2008), 269-282.

[SY] Sasaki, T. and Yoshida, M., Hyperbolic Schwarz maps of the Airy and the confluent hypergeometric differential equations and their asymptotic behaviors, J. Math. Sci. Univ. Tokyo, 15 (2008), 195-218.

[Si] Sibuya, Y., Global theory of a second order linear ordinary differential equation with a polynomial coefficient, North-Holland Mathematics Studies, Vol. 18, North-Holland, 1975.

[T] Takano, K., Ordinary Differential Equations, Asakura Shoten, Tokyo, 1994 (In Japanese).

[W] Wasow, W., Asymptotic expansions for ordinary differential equations, Interscience Publishers John Wiley \& Sons, Inc., New York-London-Sydney, 1965.

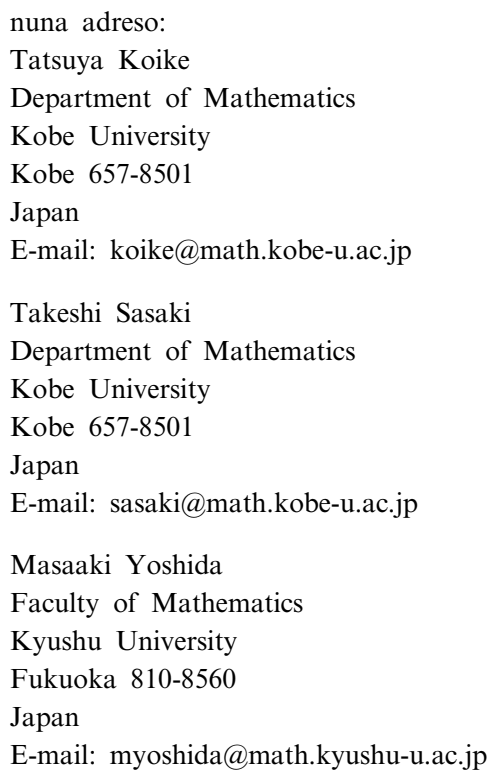

